\title{
Electroanalytical Sensor Technology
}

\author{
Aoife C. Power and Aoife Morrin
}

Additional information is available at the end of the chapter

http://dx.doi.org/10.5772/51480

\section{Introduction}

The subject of electrochemical sensors is broad, spanning many aspects of physical and analytical chemistry, materials science, biochemistry, solid-state physics, device fabrication, electrical engineering, and even statistical analysis. Thus, the field of electrochemical sensors cannot be dealt with holistically in a single chapter. Here, we will focus on electrochemical sensor technology from an analytical perspective, where the rigours of sensor behaviour will be discussed as they relate to the quality of the quantitative information that can be derived. The definition of analytical chemistry was given by the Federation of European Chemical Societies (FECS) in 1993 [1] and adopted by IUPAC:

"develops and applies methods, instruments and strategies to obtain information on the composition and nature of matter in space and time, as well as on the value of these measurements, i.e., their uncertainty, validation, and/or traceability to fundamental standards."

Electroanalytical chemistry, also known as electroanalysis, lies at the interface between analytical science and electrochemistry. It is concerned with the development, characterisation and application of chemical analysis methods employing electrochemical phenomena. It has major significance in modern analytical science, enabling measurements of the smallest chemical species, right up to the macromolecules of importance in modern biology. The history of electrochemical sensors starts basically with the development of the glass electrode by Cremer in 1906 [2]. Haber and his student Klemensiewicz took up the idea in 1909 and made the basis for analytical applications [3]. The former wanted to introduce the device as "Haber electrode" causing protests of Cremer. The latter should be given full appreciation of the invention of the glass electrode though Haber dominates the literature [4]. Today, the electrochemical sensor plays an essential analytical role in the fields of environmental conservation and monitoring, disaster and disease prevention, and industrial analysis. A typical chemical sensor is a device that transforms chemical 
information in a selective and reversible way, ranging from the concentration of a specific sample component to total composition analysis, into an analytically useful signal. A huge research effort has taken place over the several years to achieve electrochemical sensors with attractive qualities including rapid response, low cost, miniaturisable, superior sensitivity and selectivity, and appropriate detection limits. Approximately 2000 peer-reviewed papers concerning electrochemical sensors were published in 2011 according to Thomson Reuters Web of Knowledge ${ }^{\circledR}$ showing the considerable research effort underway in this field.

In the highly diverse field of chemical (and biochemical) sensing, the sensor is governed by both the aspect of the environment it is measuring and the matrix in which it is in. As well as sensors that use electrochemistry as the type of energy transfer that they detect, optical [5], thermal [6] and mass-based [7] sensors are also well-developed. From an analytical perspective, electrochemistry is appealing as it directly converts chemical information into an electrical signal with remarkable detectability, experimental simplicity and low cost. There is no need for sophisticated instrumentation, e.g., optics. A very attractive feature of electrochemistry is that it depends on a surface phenomenon, not an optical path length, and thus sample volumes can be very small, lending itself to miniaturisation.

The interest in electrochemical sensors continues unabated today, stimulated by the wide range of potential applications. Their impact is most clearly illustrated in the widespread use of electrochemical sensors seen in daily life, where they continue to meet the expanding need for rapid, simple and economic methods of determination of numerous analytes [8-10]. Through the refinement of existing sensing technologies along with the development of innovative functional sensor materials including nano- and biological materials [11-14], improved data analysis [15], and sensor fabrication and miniaturisation [16-18], opportunities for the construction of new generation sensors with much improved performances are emerging. Two branches of electrochemical sensors are developing: sensors with increased specificity and sensors capable of simultaneous/multiplex determination. In both of these branches, the ability to operate in complex biological matrixes will remain critical, forcing researchers to solve problems of biocompatibility and stability [19]. As such, the analytical and physical properties that must be considered when developing (and commercialising) chemical and biological sensors include (but are not limited to):

- Cost

- Miniaturisation

- Sensitivity

- Sensor reproducibility

- $\quad$ Selectivity/Specificity

- Multi-analyte detection

- Stability

Few sensors, if any, exhibit optimal characteristics for all properties. For example, for in vivo application, due to the complex environment of these measurements, sensing selectivity becomes critical and a trade-off between selectivity and other parameters such as cost or 
response time is needed [20]. When developing electrochemical sensors, some properties can be prioritised over others depending on final application. An electrocatalytic hydrogen peroxide sensor with a low milli-molar $(\mathrm{mM})$ detection range may, for example, have application in waste water or industrial applications. However, in order to be viable for application in clinical diagnostics, it would demand a sensitivity several orders of magnitude lower.

Following an overview of the standard sensing technologies and a brief introduction to biosensors, this chapter will review chemical and biological sensors under the criteria listed above, discussing the latest research developments in these areas published in the peerreview literature in the last three years. We will focus on all the facets of electroanalytical sensing technology with particular emphasis on the impact of nanotechnology and nanomaterials, microfabrication and biotechnology on the field to date.

\section{Electrochemical sensing principles}

Depending on the exact mode of signal transduction, electrochemical sensors can use a range of modes of detection such as potentiometric, voltammetric and conductimetric. Each principle requires a specific design of the electrochemical cell. Potentiometric sensors are very attractive for field operations because of their high selectivity, simplicity and low cost. They are, however, less sensitive and often slower than their voltammetric counterparts. In the past, potentiometric devices have been most widely used, but there is an increasing amount of research being carried out on amperometric sensors that has tipped this balance. There are also sensors relying simply on conductivity changes of ions, but there is a far larger group of sensors, which work on resistivity and impedance, such as chemiresistors and capacitive sensors. As the underlying operating principle for conductimetric sensors is usually not an electrochemical reaction or property, they should be classified as electrical chemical sensors rather than as electrochemical ones. While most electrochemical techniques for sensing analytes of interest are based on the changes in potential or current, Shan et al. [21] have developed a completely novel method for performing electrochemical measurements. In their work, they report a method for imaging local electrochemical current using the optical signal of the electrode surface generated from a surface plasmon resonance (SPR). The electrochemical current image is based on the fact that the current density can be easily calculated from the local SPR signal. The authors demonstrated this concept by imaging traces of TNT on a fingerprint on a gold substrate.

More detailed theoretical discussions on potentiometric, voltammetric and conductimetric measurements are available in this and many other textbooks [22-24] and so will not be discussed in detail here.

\subsection{Potentiometric sensors}

In potentiometric sensors, the analytical information is obtained by converting the recognition process into a potential signal, which is logarithmically proportional to the 
concentration (activity) of species generated or consumed in the recognition event. The Nernst equation logarithmically relates the measured electrode potential, E, to the relative activities of the redox species of interest:

$$
E=E^{O}+\frac{R T}{n F} \ln \frac{a^{O}}{a^{R}}
$$

Where $E^{O}$ is the standard electrode potential (quoted relative to the idealised Standard Hydrogen Electrode, SHE) and $a^{O}$ and $a^{R}$ are the activities of the oxidised and reduced species, $R$ is the universal gas constant; $T$ is the absolute temperature; $F$ is the Faraday constant; $n$ is the number of moles of electrons exchanged in the electrochemical reaction.

The most representative potentiometric sensor is the ion selective electrode (ISE)[25]. The ISE uses an indicator electrode which selectively measures the activity of a particular analyte ion. An ion-selective membrane, placed at the tip of the electrode, is designed to yield a potential signal that is selective for the target ion. This potential signal is generated by a charge separation at the interface between the ion-selective membrane and the solution due to selective partitioning of the ionic species between these two phases. The response is measured under conditions of essentially zero current. The response of the indicator electrode should be fast, reversible and governed by the Nernst equation.

In classical ISEs, the arrangement is symmetrical [26] which means that the membrane separates two solutions, the test solution and the inner solution with a constant concentration of ionic species. The electrical contact to an ISE is provided by a reference electrode (usually $\mathrm{Ag} / \mathrm{AgCl}$ ) in contact with the internal solution that contains chloride ions at constant concentration. The measured ISE potential is the sum of the two reference electrode potentials, the membrane potential constituted by boundary potentials at each membrane/solution interface, and a possible diffusion potential which may be caused by an ion concentration gradient within the ion-selective membrane phase.

ISEs with solid inner contacts are considered to be asymmetrical [26]. Taking into consideration that potentials generated at each membrane interface are included in the overall sensor signal response it is clear that to obtain a solid-contact ISE with a stable electrode potential, a fast and thermodynamically reversible ion-to-electron transduction in the solid state is required without any contribution from parasitic side reactions. Solidcontact potentiometric ion-selective electrodes have nowadays similar performance characteristics to conventional inner-solution ion-selective electrodes and offer new and advantageous technical possibilities such as miniaturization to the $\mu \mathrm{m}$ scale, cost-effective fabrication, no need for maintenance, flexibility, and multiple shape configurations. Among the electroactive materials available today, conducting polymers have emerged as a promising ion-to-electron transducers for solid-contact ISEs [25, 27, 28]. In this type of solidcontact ISE, the conducting polymer is coated with a conventional ion-selective membrane, and the ion-selectivity is determined mainly by the ion-selective membrane. For example, potentiometric sensors based on a glassy carbon electrode covered with polyaniline and various thiacalix[4]arene ionophores have been developed and applied for the successful determination of $\mathrm{Ag}^{+}$ions [29]. Other configurations for solid-contact ISEs recently reported 
involve the use of carbon cloth coated with a conventional plasticized PVC-based $\mathrm{K}(+)$ selective membrane [30], carbon nanotubes (CNTs) drop-coated with a $\mathrm{K}^{+}$-selective polyacrylic membrane [31], PVC-based molecularly imprinted polymers [32] and graphene coated with an ionophore-doped polymeric membrane [33].

ISEs are been combined with semiconductor field effect transistor (FET) technology to give ion-selective FETs, where the gate has been replaced by an ion-selective membrane. ISFETs with bare gate insulator (silicon oxide, silicon nitride, aluminium oxide, etc.) show intrinsic $\mathrm{pH}$-sensitivity due to electrochemical equilibrium between protonated oxide surface and protons in the solution. To obtain sensitivity to other ions a polymeric membrane containing some ionophore is deposited. The advantages of ISFETs include small size and rugged construction, making it a useful sensing technology for environmental and industrial analysis.

Light addressable potentiometric sensors (LAPS) [34, 35] is another type of field-effect transducer that are used as potentiometric chemical sensors which has gained reasonable popularity. Their principle of operation is quite similar to that of ISFETs in which the drainsource current in a space charge region at the semiconductor/insulator interface depends on the applied gate potential. Illumination by light source with modulated intensity generates an AC photocurrent that depends on the applied potential. Like ISFET sensors, ion-selective membranes of various types may be deposited onto the insulator surface to give the required ion sensitivity [26].

\subsection{Voltammetric sensors}

Voltammetry provides an electroanalytical method, the premise of which is that current is linearly dependent upon the concentration of the electroactive species (analyte) involved in a chemical or biological recognition process (at a scanned or fixed potential). Voltammetry implies a varying voltage. Cyclic voltammetry, squarewave and stripping voltammetry are some of the more common techniques. Amperometry is strictly a sub-class of voltammetry in which the electrode is held at constant potentials for various lengths of time. The distinction between amperometry and voltammetry is mostly historic as there was a time when it was difficult to switch between "holding" and "scanning" a potential. This function is trivial for modern potentiostats, and today there is little distinction between the techniques which either "hold", "scan", or do both during a single experiment. The recent review by Gupta et al. [36] focuses on the various applications of voltammetry to pharmaceutical analysis from 2001 to 2010.

Stripping analysis is one of the most sensitive voltammetric methods [37, 38]. Such techniques enjoy the advantages that there is no need for derivatization and that these methods are less sensitive to matrix effects than other analytical techniques. It is mostly used for trace analysis of heavy metals for environmental analysis [39, 40]. Cathodic or anodic stripping voltammetry have also been used for a highly sensitive determination of nucleobases, nucleosides, nucleotides or acid-hydrolyzed NAs, based on formation of sparingly soluble complexes of the NA constituents with electrochemically generated mercury or copper(I) ions [41]. 
In amperometry, the working electrode is held at a constant potential while the current is monitored. The current is then related to the concentration of the analyte present. This sensing method is commonly employed in both biosensors and immunosensors, which will be discussed further later. The first amperometric sensor was the oxygen electrode developed by L.C. Clark [42]. Oxygen entering the system through a gas-permeable membrane is reduced to water at a noble metal cathode. Clark also described the first glucose biosensors in 1962, using his oxygen electrode to determine the depletion of oxygen by the action of glucose oxidase on glucose [43]. Today, about 50 years on, Hu et al. [44] reported an oxygen sensor based on an inkjet printed nanoporous gold electrode array on cellulose membranes using ionic liquid as electrolyte. The sensor looks like a piece of paper but possesses high sensitivity for $\mathrm{O}_{2}$ in a linear range from 0.054 to $0.177 \mathrm{v} / \mathrm{v} \%$. Hydrogen peroxide sensors as well as non-enzymatic glucose sensors are of particular interest in the field of amperometric sensors [20].

The development of all voltammetric techniques has been based predominantly on the use of mercury, carbonaceous materials and noble metals as working electrodes. However, toxicity of mercury, inconvenience in working with liquid hanging drop electrodes, and a limited range of potentials for mercury for anodic reactions has essentially eliminated mercury from this list. Carbon-based working electrode materials include all allotropic forms of carbons - graphite, glassy carbon, amorphous carbon, fullerenes, nanotubes, and are all used as important electrode materials in electroanalytical chemistry. Over the past five decades, carbon paste, i.e., a mixture of carbon (graphite) powder and a binder (pasting liquid), has arguably become one of the most popular carbon electrode materials used for sensors given that carbon pastes can readily be screen-printed for the mass-production of electrodes. Screen-printing is a thick-film process, which has been used for many years in artistic applications and, more recently, for the production of miniature, robust and cheap electronic circuits. During the early 1980s, the process was adapted for the production of carbon-paste-based amperometric biosensors which had a huge impact on biosensor commercialisation. The majority of most successful electrochemical sensors, including the blood glucose biosensor strip, to date, employ a screen-printed carbon-paste as the working electrode $[45,46]$.

The use of new materials, especially nanomaterials, has become an increased area of research in electrochemical sensors. The incorporation of these nanomaterials in conjunction with one another to form novel composites is particularly interesting, as many of these materials have been found to have synergistic effects. Zhong et al. [47] reported a nonenzymatic hydrogen peroxide amperometric sensor based on a glassy carbon electrode modified with an MWCNT/polyaniline composite film and platinum nanoparticles. The composite films have a large specific surface, high redox electrochemical activity and good environmental stability. Due to the synergistic catalytic activity between MWCNTs-PANI nanocomposites and $\mathrm{Pt}$ nanoparticles, the fabricated non-enzyme amperometric sensor displayed a high sensitive detection of $\mathrm{H}_{2} \mathrm{O}_{2}$. CNTs were also used by Guo et al. [48] who developed a sensitive amperometric sensor for tryptophan by modifying a glassy carbon (GC) electrode with gold nanoparticle decorated CNTs. The nanocomposite material 
demonstrated synergistic enhancement for the electrocatlytic activity toward the oxidation of tryptophan.

\subsection{Conductimetric and impedimetric sensors}

Conductimetric sensors are based on the measurement of electrolyte conductivity, which varies when the cell is exposed to different environments. The sensing effect is based on the change of the number of mobile charge carriers in the electrolyte. If the electrodes are prevented from polarizing, the electrolyte shows ohmic behaviour. Conductivity measurements are generally performed with an AC supply. The conductivity is a linear function of the ion concentration; therefore, it can be used for sensor applications. Ventura et al. [49] have reported a paper-based conductivity sensor for hydrogen. Palladium nanoparticles were attached onto a covalently cross-linked CNT paper. Depending on the design of the sensor, p-type or n-type conduction was observed as the resistance of the paper was measured under exposure to hydrogen gas. This paper demonstrates a simply designed, flexible gas sensor, highlighting the next stage in the development of functional nanotube paper. Another format reported was an electrospun nylon 6,6 nanofiber containing an ionic liquid (1-butyl-3-methylimidazolium hexafluorophosphate) was electrospun and found to behave as an effective, reversible chemiresistor for detecting organic vapours, highlighting another route to a simple, flexible gas conductivity sensor [50]. Conductivity measurements in general, are non-specific for a given ion type. Also, the polarization and the limiting current operation mode need to be avoided as these can damage the electrode interface. Thus, a small amplitude alternating bias can be used for sensor measurements with frequencies where the capacitive coupling is still not determining the impedance measurement. This was used for the sensing of ammonia on polyanilinemodified interdigitated arrays to prevent polarisation of the polyaniline [51].

Impedance-based sensors have a similar design as mixed potential type sensors. Instead of measuring the voltage, a sinusoidal voltage is applied and the resulting current is measured. Impedance is then calculated as the ratio of voltage to current in the frequency domain. By using small amplitude sine wave perturbation, linearity in electrochemical systems can be assumed, allowing frequency analysis. An excellent review of the use of impedance for the sensing of gases has recently been published by Rheaume \& Pisano [52].

The use of impedance/capacitance has also been used to detect the antibody/antigen complex formation which has application in electrochemical immunosensors. This phenomena was first reported in 1998 when upon formation of an Ab/Ag complex on the surface of the electrode, the increase in dielectric layer thickness caused changes in capacitance proportional to the size and the concentration of antibodies [53]. Impedance changes between electrode surfaces and a surrounding solution upon a binding event can be transduced into an electrical signal using a frequency response analyser. There are several theories as to how this binding event affects changes in real and imaginary components of the system, although it is difficult to identify the origin of these changes. One theory hypothesises that binding of larger antigens forms a resistive barrier, causing the impedance to increase whilst binding of smaller antigens can 
facilitate a charge transfer and lower impedance [54]. Future work must establish the origin of this impedance change, whether from increases in surface density or perhaps from conformational changes that modify charge transfer across the sensor interface. Recent examples of impedance-based immunosensors include a silicon nitride $\left(\mathrm{Si}_{3} \mathrm{~N}_{4}\right)$ surface with covalently bound anti-human serum albumin (anti-HSA) [55], Electrochemical impedance spectroscopy (EIS), measurements for the specific detection of HSA proteins where a detection limit of $10^{-14} \mathrm{M}$ were achieved. This is one of a handful of papers that have generated EIS measurement on insulating surfaces. Mostly impedimetric immunosensors are based on conducting materials. Chemically modified graphene (CMG), immobilised on a printed electrode was demonstrated as a disposable platform for the attachment of anti-IgG (antiImmunoglobulin) [56]. The principle of detection lies in the changes in impedance spectra of a bulk solution-based redox probe $\left(10 \mathrm{mM} \mathrm{K}_{4}\left[\mathrm{Fe}(\mathrm{CN})_{6}\right] / \mathrm{K}_{3}\left[\mathrm{Fe}(\mathrm{CN})_{6}\right]\right)$ after the attachment of $\operatorname{IgG}$ to the immobilized anti-IgG. The immunosensor was optimised and it was found that thermally reduced graphene oxide has the best performance in terms of sensitivity when compared to other CMG materials.

\section{Biosensors}

Biosensors aim to utilise the power of electrochemical techniques for biological processes by quantitatively producing an electrical signal that relates to the concentration of a biological analyte. The relatively low cost and rapid response of these sensors make them useful in a variety of fields including healthcare, environmental monitoring, and biological analysis among others [19]. Biosensors use biomolecules as recognition elements which must be immobilised (or coupled) to the transducer. The transducer is the electrode (or set of electrodes) in the case of the electrochemical sensor. The specificity and selectivity that a biosensor provides is attributed to this immobilised biological recognition element. Biocatalytic and bioaffinity recognition elements are the two classes of molecules employed. Enzymes, which are biocatalytic recognition elements are the best known and studied. Recent development has focused on improving the immobilization and stability of the enzymes [57, 58]. Enzymes immobilized to nanosized scaffolds such as spheres, fibres and tubes have all recently been reported [59-61]. The premise of using nanoscale structures for immobilization is to reduce diffusion limitations and maximize the functional surface area to increase enzyme loading. In addition, the physical characteristics of nanoparticles such as enhanced diffusion and particle mobility can impact inherent catalytic activity of attached enzymes [62]. Increased enzyme stability at these surfaces is also widely reported. A novel platform based on buckypaper (which is a thin membrane of CNT networks) was reported using glucose oxidase as the model enzyme. A biocompatible mediator-free biosensor was studied and the potential effect of the buckypaper on the stability of the biosensor was assessed. The results showed that the biosensor had a considerable functional lifetime of over 80 days [58]. Greatly enhanced electrochemistry of the enzyme can be observed by the use of nanoparticles, due to the ability of the nanoparticles to reduce the distance between the redox centre of the enzyme and the electrode. Willner's group has contributed significantly to this area by taking the approach of reconstituting apo-proteins on cofactor-modified electrodes as a general strategy to electrically contact redox enzymes with electrodes $[63,64]$. 
A recent news article in Nature Chemistry [65] points to the major Achilles heel of enzymebased sensors. To measure glucose, enzymes such as glucose oxidase, hexokinase, or glucose dehydrogenase can be coupled to reactions that generate a signal. But such an analytical setup can be used only if analogous enzymes are available for the analyte of interest. They identify a paper by Lu \& Xiang [66] that overcomes this severe limitation where a method (Figure 1) for expanding the principle of the glucose meter to detect other analytes is reported. Instead of measuring the analyte directly, their strategy aims to produce glucose in quantities proportional to the amount of the analyte of interest. The approach uses magnetic microbeads coated with an enzyme (invertase) that is conjugated to either DNA aptamers or DNAzymes. These DNA oligomers can be selected from huge libraries of random DNA sequences, based on their binding to an analyte of choice. When the target binds to the aptamer or DNAzyme, invertase is released into the solution. After magnetic separation of microbeads, the invertase hydrolyses sucrose into glucose, which can then be quantified using a conventional glucose meter. Lu and Xiang demonstrate the measurement of four different classes of non-glucose analytes: a small molecule (cocaine), a nucleoside (adenosine), a protein (interferon-gamma) and a metal ion (uranium).

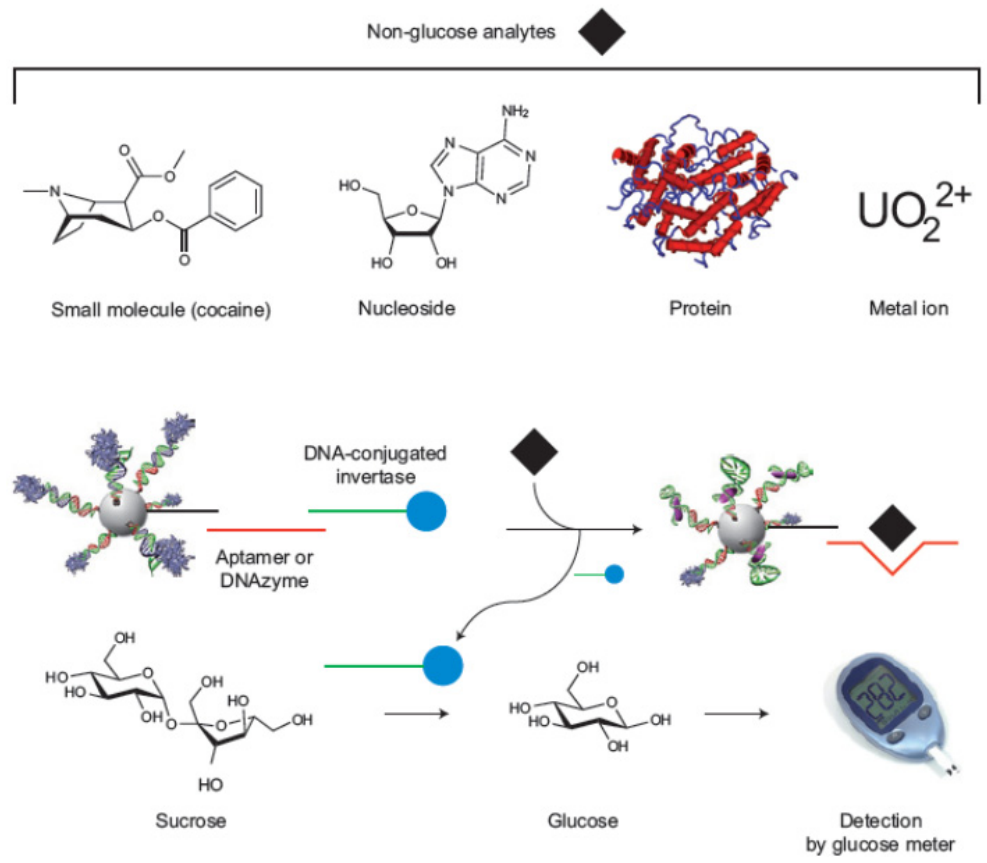

Figure 1. Sensing a variety of analytes using a glucose meter. Aptamers or DNAzymes that bind a wide range of analytes can be selected from huge libraries of random DNA sequences. When the analyte binds to its aptamer, invertase is released. Sucrose is then converted to glucose in proportion to the original analyte and can be detected using a standard glucose meter. Reprinted by permission from Macmillan Publishers Ltd: [Nature Chemistry] (Sia SK and Chin CD, Analytical Chemistry: Sweet Solution to Sensing, Nature Chemistry, 3: 659-660.), copyright (2011). 
The second class of bioaffinity recognition elements such as antibodies and DNA are also widely used in biosensing. Immunosensors, which perform immunoassays based on antigen and antibody recognition, have become vital for the determination of biochemical targets relating to health concerns spanning from cancer antigens [67] to pathogens [68]. Some of the most significant advances include development of immunosensors for the continuous monitoring of analytes, point of care (PoC) devices, with lower unit costs, automation, reusability and ease of use [8]. A continual concern with immunosensor development is the capability to sensitively detect relevant immunological compounds without compromising the bioactivity of the immunoactive species on the electrode. Stabilisation of sensor constructs was achieved by using polylysine films into which antibiotin molecules were immobilised [69]. Cross-linking prevents conformational change and unfolding of the antibodies allowing markedly enhanced sensitivity when compared with similar constructs, longer storage times and higher resistance to extremes of $\mathrm{pH}$ and temperature. Many researchers have increasingly utilized nanomaterials to support the immunoactive agents while simultaneously enhancing the electrochemical and analytical capabilities of the electrode [70, 71]. A triple signal amplification strategy was designed by Lin et al. [17] for the ultrasensitive immunosensing of a cancer biomarker, carcinoembryonic antigen (CEA). This strategy was achieved using graphene for accelerating electron transfer, poly(styrene-co-acrylic acid) microbead (PSA) carried Au nanoparticles (NPs) as tracing tags to label signal antibody and Au NPs induced silver deposition for anodic stripping analysis. An in situ synthesis of Au NPs led to the loading of numerous $\mathrm{Au}$ NPs on the PSA surface and convenient labelling of the tag to the secondary $\mathrm{Ab}$. With the sandwich-type immunoreaction, the anchored Au NPs further induced the chemical deposition of silver for electrochemical stripping analysis of target antigen. The triple signal amplification greatly enhanced the sensitivity for biomarker detection. A detection limit as low as to $0.12 \mathrm{pg} / \mathrm{mL}$ was reported. The immunosensor exhibited good stability and acceptable reproducibility and accuracy, indicating potential applications in clinical diagnostics.

In recent years there has been increasing interest in finding new molecules that are able to mimic antibodies and replace them for therapeutic purposes and for bioanalytical applications. Antibodies are difficult and expensive to manufacture and are produced in vivo, by immunizing animals. Different molecules are currently being studied as alternatives to antibodies for bioanalytical applications; among these are nucleic acid aptamers. One of the main advantages of nucleic acid aptamers compared with antibodies is their in vitro selection procedure and their chemical synthesis. These manufacturing procedures do not depend on a particular analyte (possibility of using toxins, and molecules that do not elicit a good immune response) and enable the use of non-physiological conditions (including extremely high or low temperature and $\mathrm{pH}$ ), do not require animals and cell lines, and are cost-effective. Most of the electrochemical detection principles described for other bioaffinity assays are applicable to electrochemical aptamer-based biosensors, or aptasensors. Label-free modes are based on the change in electrode surface behaviour after formation of the aptamer-target complex (usually monitored by electrochemical impedance spectroscopy $[19,72]$ or by use of a FET [73]. 


\section{Physical and analytical properties of electroanalytical sensors}

\subsection{Cost}

Electrochemical sensors provide a low cost analytical tool. Furthermore, the ability to produce electrochemical sensors in large numbers at a low cost is a major requirement for many applications. For instance, for commercial sensors aimed at the medical self-testing and smart packaging markets, single-shot use has many advantages as it immediately overcomes issues with cross-contamination and integrating sensors into disposable packaging. Thus, the ability to mass manufacture sensors, with minimal production costs facilitates the potential of sensors as low-cost disposable platforms.

The current state-of-the-art in sensor manufacturing is the commercial glucose test strip which is produced in volumes of billions annually. Given the volumes being manufactured, the cost per sensor is a fraction of a cent. The manufacturing technology that has been exploited so successfully for this is screen-printing [46]. Screen-printing is a thick-film process, which has been used for many years in artistic applications and, more recently, for the production of miniature, robust and cheap electronic circuits. During the early 1980s, the process was adapted for the production of amperometric biosensors which had a huge impact on biosensor commercialisation. The majority of most successful electrochemically based devices, including the blood glucose biosensor strip, to date, have used screen-printing as the manufacturing tool [74]. Another type of low-cost printing is also being adopted for sensor fabrication is inkjet [44, $75,76]$. It has the required levels of flexibility, resolution and scale-up required for sensor production. It remains to be seen however if it is adopted by the electrochemical sensor community. Crooks research group recently reported a paper-based electrochemical sensing platform with integral battery and electrochromic read-out [77] (Figure 2). The platform is fabricated based on paper fluidics and uses a Prussian blue spot electrodeposited on an indium-doped tin oxide thin film as the electrochromic indicator. Although this is not a printed platform, the concept of using screen- and/or inkjet-printing to mass produce such a sensor could be easily envisaged. It is likely however, that a combination of print and possibly non-print methods (e.g., photolithography) will be adopted in the future in order to produce systems comprising not only the sensor component, but also the display, power and circuitry components in order to build highly sophisticated, low-cost autonomous systems [45].

\subsection{Miniaturisation}

Through the reduction in size of both the functionally critical components of sensing devices and the sensors themselves, miniaturisation offers a range of distinct advantages to the analyst, including the reduction of:

- $\quad$ transport times and volumes

- dead volumes

- sample preparation

- reagent consumption

- energy consumption

- time expenditure and monetary cost. 


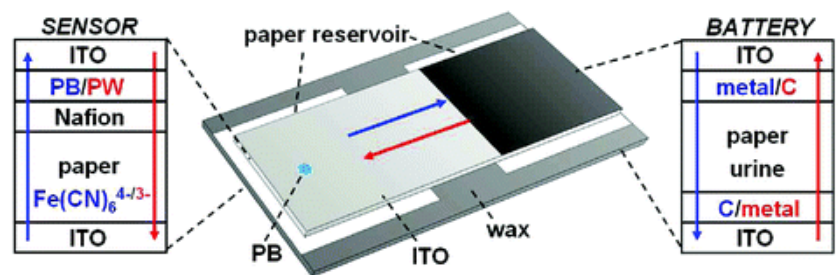

$$
\begin{gathered}
\text { glucose+Fe(CN) }{ }_{6}{ }^{3-\mathrm{GOX}} \underset{-}{\mathrm{Ge}(\mathrm{CN})_{6}{ }^{4-}} \longrightarrow \text { current for glucose sensing } \\
\mathrm{H}_{2} \mathrm{O}_{2}+\mathrm{Fe}(\mathrm{CN})_{6}{ }^{4-} \stackrel{\mathrm{HRP}}{\longrightarrow} \mathrm{Fe}(\mathrm{CN})_{6}{ }^{3-} \quad \longrightarrow \text { current for } \mathrm{H}_{2} \mathrm{O}_{2} \text { sensing }
\end{gathered}
$$

Figure 2. Drawing illustrating the operational principles of a paper-based electrochemical sensing platform with integral battery and electrochromic read-out. The device consists of two parts: a sensor section and an $\mathrm{Al} /$ air battery section, which are separated by a wax barrier. For glucose detection, the paper reservoir of the sensor section is preloaded with dried $\mathrm{GOx}$ and $\mathrm{Fe}(\mathrm{CN}) 6^{3-}$. The catalytic oxidation of glucose by $\mathrm{GOx}$ results in conversion of $\mathrm{Fe}(\mathrm{CN}) 6^{3-}$ to $\mathrm{Fe}(\mathrm{CN}) 6^{4-}$. $\mathrm{Fe}(\mathrm{CN}) 6^{4-}$ is then oxidized back to $\mathrm{Fe}(\mathrm{CN}) 6^{3-}$ on the lower ITO electrode, which results in reduction of PB to colorless PW on the upper ITO electrode. The $\mathrm{H}_{2} \mathrm{O}_{2}$ sensor operation is similar, except that $\mathrm{HRP}$ catalyzes the oxidation of $\mathrm{Fe}(\mathrm{CN}) 6^{4-}$ to $\mathrm{Fe}(\mathrm{CN})_{6^{3-}}$ in the presence of $\mathrm{H}_{2} \mathrm{O}_{2}$. The resulting $\mathrm{Fe}(\mathrm{CN}) 6^{3-}$ is then reduced at the lower ITO electrode, while PW is oxidized to PB at the upper ITO electrode. The battery section of the device drives the electrochemical reactions in the sensor section [77]. Reprinted with permission from (Liu H and Crooks RM, Paper - Based Electrochemical Sensing Platform with Integral Battery and Electrochromic ReadOut, Analytical Chemistry, 84: 2528-2532). Copyright (2012) American Chemical Society.

This coupled with improved portability has resulted in miniaturisation becoming a major driving force in sensor research, prompting not only the scaling down of established sensing devices [78], but also the development and application of novel sensing materials $[79,80]$ in a variety of sensors. At present the majority of electrochemical sensor development is application orientated which has resulted in the extreme miniaturisation of sensing devices. This is in a large way due to the concurrent advances in nanotechnology which has allowed the fabrication of novel sensors possessing signal transduction mechanisms that exploit the unique physical phenomena of the nanoscale [81]. These sensors may utilise, for example, the spectroscopy of plasmonic nanoparticles, the deflection of cantilevers and the conductivity of nanowires [81,82].

The focus of this section will be on the development of novel sensors produced through novel fabrication techniques, which show comparable or improved sensing performance with conventional sensors while also highlighting improvements in the ideal sensor features listed in the chapter's introduction. Success in terms of the improved selectivity and stability of miniaturised potentiometric sensors will be further discussed in following sections.

The impact miniaturisation is having on the field of electrochemical research is most readily visualised when we consider the development of biosensors, specifically those that are commercially available, the most prominent of which is the glucose sensor. In his review Electrochemical Glucose Biosensors, Wang, describes in detail the refinement of the original glucose sensor fabricated by Clark and Lyons in 1962, to today's commercially available disposable electrodes with portable personal readout devices to the potential, continuous non-invasive in vivo real time monitoring [83]. 
Continuing the theme of non-invasive in vivo monitoring, Wang also describes the development of a miniaturised flow cell amperometric sensor, which could be inserted into a subjects tear duct. The screen printed microflow detector was first shown to possess the ability to detect catecholamine neurotransmitters norepinephrine and dopamine with the rolled microsensor giving a rapid and sensitive responses to trace levels of the two neurotransmitters (approx. $165 \mathrm{nM}$ norepinephrine and $530 \mathrm{nM}$ dopamine). Further functionalization of the sensor by the addition of a carbon working electrode coated with a film containing glucose oxidase, allowed the sensitive detection $(8 \mu \mathrm{M})$ of glucose [84].

Wang's group also depict their work with screen printed electrode (SPE) as sensors. Screen printing is a technique that offers a variety of advantages including low-cost mass production, minimal sample volume and low cross-contamination [85]. Au SPEs, coated with a ternary monolayer interface, containing hexanedithiol (HDT), SHCP a thiolated capture probe, and 6-mercapto-1-hexanol $(\mathrm{MCH})$ were shown to offer direct, sensitive detection of nucleic acid hybridization in serum and urine. Modification of the sensing layer further improved the sensors sensitivity with a 10-fold improvement in the signal-to-noise ratio for a $1 \mathrm{nM}$ target DNA compared to common SHCP/MCH binary interfaces. The SPEs also allowed direct quantification of the target DNA down to $25 \mathrm{pM}$ and $100 \mathrm{pM}$ in undiluted serum and urine samples, respectively. These SPEs possessed good anti-fouling properties after extended exposure to raw human serum and urine samples, showing good potential as low cost nucleic acid sensors [86].

In another account Wang et al. outline their development of wearable SPEs for the voltammetric detection of trace heavy metal contaminants (copper linear detection from 10 $90 \mathrm{ppb}$ with a limit of detection (LOD) of $13 \mathrm{ppb}$ ) and nitro - aromatic explosives (detection range of $100-900 \mathrm{ppb}$ of TNT with a LOD of $42 \mathrm{ppb}$ ) in seawater [87]. The modification of SPEs by inkjet printing is further illustrated by a number of other groups including Sriprachuabwong et al [88] who describe how the graphene-PEDOT:PSS modified SPEs electrochemical activity to $\mathrm{H}_{2} \mathrm{O}_{2}$, nicotinamide adenine dinucleotide (NADH) and $\mathrm{K}_{4} \mathrm{Fe}(\mathrm{CN})_{6}$ is significantly enhanced, in comparison to unmodified SPEs.

Microelectrodes are another popular means of miniaturisation as they exhibit increased temporal resolution and current densities, with reduced ohmic drop and charging currents, and high Faradaic to capacitive current ratios. However individually their current output is often overshadowed by electrochemical noise. To counteract this, microelectrode arrays are fabricated, improving sensitivity and facilitating lower detection limits in comparison to macroelectrodes [89]. Thus microelectrodes are one of the most obvious successes in sensor miniaturisation because they are small, able to operate in low sample volumes and with portable instrumentation systems. This has resulted in a significant volume of research into the working mechanism of microelectrodes particularly as they do not experience an ohmic drop during their operation [90-92].

Although the continuous improvement of microfabrication techniques has enabled researchers to fabricate ultramicroelectrodes of increasingly diminutive size [93] it is through the incorporation of novel materials that the greatest advances in sensor 
development are seen. For example, Dumitrescu et al. describe the fabrication of ultramicroelectrodes composed of networks of single-walled CNTs using lithography. The resulting electrodes exhibited low background current and decreased double layer charging in comparison to macroelectrodes, which coupled with their low surface area and capacitance resulted in fast response times in comparison to metal ultramicroelectrodes making them suitable for a variety of applications [94]. Similarly other groups report the application of printed electrodes in environmental and biological analysis $[95,96]$

The work of Banks et al. highlights the advantages of miniaturised SPEs over conventional carbon electrodes, specifically in terms of cost and time efficiency. SPEs have been shown to possess a greater ease of use, scale of economies and are disposable in nature, requiring reduced volumes of the analyte $(\mu \mathrm{l})$. This gives them a significant advantage over conventional (solid) carbon electrodes and other fabrication approaches (such as lithography) in sensing applications. The incorporation of nanomaterials to improve electron transfer processes, selectivity and sensitivity will only further enhance the field of electrochemical sensors pushing towards smaller and well-defined geometries in formats such as screen printed microelectrode arrays and 'lab on a chip' approaches with the overall ethos of simpler, cheaper, disposable, scalable and ease of use [85].

It follows that the application of interdigitated electrode arrays (IDAs) can also exploited to further miniaturise sensing systems often through the incorporation of nanocomposites, Liu et al. [97] describes the detection of volatile sulphur compounds (associated with a variety of ailments [98-100]) in a subject's breath exhalations. The authors report that the fabricated PANI/AuNPs sensor electrodes exhibited a good response to both $\mathrm{H}_{2} \mathrm{~S}(1.0 \mathrm{ppm})$ and $\mathrm{CH}_{3} \mathrm{SH}$ (1.5 ppm) gases and that in comparison with other sensors, these novel IDA electrodes possess a greater selectivity and sensitivity while also being low cost. IDAs were also the basis of a humidity sensor developed by Cassidy et al. [101] where an Ag polymer nanocomposite was shown to give linear detection over a range of $10-60 \%$ relative humidity $(\mathrm{RH})$, the resulting sensor was found to be reversible and repeatable with minimal hysteresis.

The design and fabrication of electrochemical sensors remains a vibrant area of research with the miniaturization of electrodes continuing down to the nano regime, allowing the routine measurement of fast electron kinetics at diminutive analyte concentrations. The use of nanomaterials in sensor design continues to increase enabling significant improvements in the analytical performance and utility of biosensors.

\subsection{Sensitivity}

The sensitivity of a sensor is defined as the slope of the analytical calibration curve for a given analyte. A sensor is sensitive when a small change in analyte concentration causes a large change in the response. Within the linear range of response, the sensitivity is a welldefined value. Improvements in sensitivity of sensors have always been of paramount interest [102]. Immunoassays based on specific antigen-antibody recognition, is a promising 
approach for selective and sensitive analysis. Electrochemical immunosensors, which combine specific immunoreactions with electrochemical transduction, have attracted growing attention in recent years. They have many advantages including simple instrumentation and operation, high sensitivity and selectivity, and wide linear range. Since enzyme labels provide great signal amplification in the assay and also a large number of antibody-enzyme or antigen-enzyme conjugates are commercially available, the majority of electrochemical immunoassays are based on the use of specific enzyme/substrate couples [103-105]. Sensitivities of $\mathrm{ng} / \mathrm{ml}$ can routinely be achieved in enzyme-based electrochemical immunosensors. Many interesting signal amplification strategies have been adopted to further improve sensitivity of these sensors [106-108]. For example, an immunosensor with a detection limit of $1.0 \mathrm{pg} / \mathrm{mL}$ was developed for Interleukin-6 (IL-6) based on a dual amplification mechanism resulting from Au nanoparticles (AuNP)-poly-dopamine (PDOP) as the sensor platform and multienzyme-antibody functionalized AuNP-PDOP CNT. Nonenzymatic immunosensors have also been reported to be able to achieve very low detection limits. Pichetsumthorn et al. [102] reported a nonenzymatic impedimetric biosensor for the small molecule atrazine. They used nanoporous alumina membranes and interfaced them with a printed circuit board. The assay achieved non-faradic detection, i.e. the transfer of charge between the biomolecule and the electrode is mediated without the use of a redox probe. The limit of detection was in the $\mathrm{fg} / \mathrm{mL}$ regime with a dynamic range of detection spanning from $10 \mathrm{fg} / \mathrm{mL}$ to $1 \mathrm{ng} / \mathrm{mL}$. Such high sensitivity was attributed to the confinement of the small molecules in the nanopores of the alumina membranes which resulted in an amplification of the impedance signal measured due to the modulation of the electrical double layer. Another highly sensitive, nonenzymatic electrochemical immunosensor [109] was constructed using a graphene platform combined with mesoporous PtRu alloy as a label for signal amplification. A low detection limit of 9.63 $\mathrm{pg} / \mathrm{mL}$ using a sandwich-type assay approach for microcystin-LR (MC-LR) was reported. Again, this approach does not rely on enzymes or electrochemical redox mediators to function, but rather on the strong catalytic action of NP-PtRu alloys upon $\mathrm{H}_{2} \mathrm{O}_{2}$. Anodic stripping voltammetry (ASV) is a highly sensitive method for the analysis of trace concentrations of electroactive species in solution. Detection limits for metal ions at sub-ppb concentrations have been reported. ASV detection of metal nanoparticle labels have been used in both immunosensors [110] and DNA sensors [111]. Au nanoparticles are the most frequently used among all the metal nanoparticle labels available. Recently, Shen \& Zhang reported a sensitive immunosensor using ASV for the detection of hepatitis B surface antigen (HBsA) based on copper-enhanced Au nanoparticle labels [112]. A linear range of 0.1-1500 ng/mL with a detection limit of $87 \mathrm{pg} / \mathrm{mL}$ was reported which was based on the determination of $\mathrm{Cu}$ by ASV.

In the past few years, many efforts have been devoted to improve the sensitivity of metal oxide gas sensors [13]. Sakai et al. found that the porous structure of the sensing film played a critical role in the performance of the sensor because it dictated the rate of gas diffusion [113]. Indeed, several groups have found that the particle size of the metal oxide heavily affected the sensitivity of sensor $[114,115]$. Although many methods have been reported to 
synthesize nanoparticles of metal oxides, often they are not stable and may easily aggregate. Besides, nanoparticles may be compactly sintered together during the high temperature film coating process, rendering them nonporous, which is a disadvantage for gas diffusion through them. If porous nanostructures are used as gas sensing materials, the gas sensing properties will be much improved. On the basis of this, nanostructures with many kinds of shapes such as porous nanowires, porous nanotubes and porous nanospheres have been reported to have excellent gas sensitivity properties. A nanocomposite of $\mathrm{SnO}_{2}$ and multiwalled carbon nanotubes (MWCNT) [116] was exploited to detect persistent organic pollutants (POPs) which possess stable chemical properties and are ordinarily difficult to detect with metal oxides [116]. The ultrasensitive detection of aldrin and dichlorodiphenyltrichloroethane (DDT) was carried out using the nanocomposite of small $\mathrm{SnO}_{2}$ particles and MWCNTs. The nanocomposite showed a very attractive improved sensitivity compared with a conventional $\mathrm{SnO}_{2}$ sensor. A LOD of $0.5 \mathrm{ng}$ was achieved for aldrin and DDT, which was attributed to the highly porous 3D structured $\mathrm{SnO}_{2} / \mathrm{MWCNT}$ nanocomposites (which enhances gas diffusion).

\subsection{Selectivity}

Selectivity is defined as the ability of a sensor to detect one specific species even in the presence of a number of other chemical species or interferents. All electrochemical sensors exhibit a high degree of selectivity. This section will discuss recent research to develop highly selective electrochemical sensors and efforts to improve the selectivity of existing sensors through the incorporation enhanced sensing membranes. This section is divided in two parts focusing first on potentiometric and then voltammetric and amperometric sensors.

\subsubsection{Potentiometric sensors}

As discussed earlier, there are several basic types of potentiometric devices including ISEs, FETs $[117,118]$. The ISE is the most representative potentiometric sensor; recently there has been a marked increase in the demand for miniaturised ISEs as interest in the biological application of potentiometric sensors. Recent advances in nanoscale potentiometry were discussed by Bakker and Pretsch with the authors discussing the nature and role of interfacial films on both sides of the ion-selective membrane and summarising the improved general performance of the nano-electrodes in terms of detection limits, biocompatibility, and sensor stability [119]. The application of conducting polymers in potentiometric sensing is discussed widely throughout the literature [120-123] with Faridbod et al. [124] reporting the use of conducting polymer-based ISEs to over 60 inorganic ions.

Ultimately the development of new means of ion recognition continues to be the main focus of ISE research, with researchers discussing the synthesis of a range of ionophores targeting silver [125, 126], lead [127] and cobalt [128] among others. In each instance improved selectivity is reported coupled with favourable LODs. Buhlmann's group having reported 
the success of highly fluorinated liquid phases as sensing materials, detailing the design of numerous ion-selective sensors which incorporate fluorinated membranes [129-131].

Throughout sensor research as a whole, the area with the greatest level of continued consistent development is that which exploits nanomaterials and their novel properties, illustrated by the rapidly growing number of publications in this area. This phenomenon is most evident in the area of electrochemical sensors. Within the field of potentiometric sensors and specifically the design of contacts for all solid state sensors, the potential of nanomaterials such as CNTs and fullerenes has been rigorously examined. Rius et al. [132135] described the development of many sensors based on SW- and/or MW- CNT sensors, including an all-solid-state potassium ion-selective membrane electrode and a perchlorate selective membrane electrode using a MWCNT-derived solid contact.

Recent advancements in the field of potentiometric sensors and especially in terms of ISEs have seen a significant focus on the development and fabrication of sensing membranes particularly in terms of their composition, where the incorporation of CNTs and unique ionophores in both carbon paste electrodes and polyvinylchloride (PVC)-based membranes. Carbon pastes utility as the basis of a sensing membrane is becoming increasingly clear, with the development of a number of sensors incorporating MWCNTs and specific ionophores [136-138].

Trace analysis has always been a strength of electrochemical detection. At present the detection of copper ions has become a priority of industry as many biochemical processes carried out in industrial settings depend on the presence of copper ions. Copper can displace metal ions in vivo and the potential of sensing techniques to determine the concentration of copper in a sample are both costly and time-consuming, prompting the fabrication of selective sensors by a number of groups [16, 139, 140]. For example, Petkovic et al. [140] discuss the development of a novel $\mathrm{Cu}^{2+}$ ISE using a PVC matrix containing $\mathrm{N}, \mathrm{N}^{\prime}, \mathrm{N}^{\prime \prime}, \mathrm{N}^{\prime \prime \prime}$-tetrakis(2-pyridylmethyl)-1,4,8,11-tetraazacyclotetradecane as the ionophore. A simple construction method affords a cost-effective, rapid measurement of copper in a mixed solution with EDTA. With the additional benefits of a superior working $\mathrm{pH}$ range and longer sensor lifetime coupled with a comparable measurement signal in comparison with commercial sensors.

Improved composition of ISE membranes has enhanced the response time for ionic sensing and as detailed above, PVC [140] has proven to be suitable for ISE construction as it allows for ease of construction and improved lifetime/robustness of the sensing matrix. PVC containing ISEs have been utilised in range of industrial studies including medical research and water contamination experiments [137, 141-144]. PVC membranes containing ISEs, have also demonstrated potential as anion selective electrodes. For example, Alvarez-Romero et al. [145] discuss the successful development of a graphite-based composite containing epoxy resin, doped with chloride ion doped polypyrrole, as a chloride ion selective electrode. Zahran et al. [146] report a novel PVC-based sensor that exhibit selectivity toward halides (chloride and bromine specifically). Similarly Kang et al [147] describe the fabrication and characterisation of a PVC based $\mathrm{Sc}^{3+}$ octaethylporphyrin composite fluoride selective 
electrode this is significant as fluoride selective sensors allow for rapid detection of toxic organic fluorophosphates, which can contaminate drinking water.

The sensing of uncharged molecules is a constant challenge for researchers as they are not influenced by ionophores traditionally used to sense anionic or cationic species. The use of molecularly imprinted polymers (MIPs) has been greatly increasing in ISEs [148-151]. For example, Liang et al. [148] report the use of a polymeric membrane ISE to detect neutral molecules, utilising a MIP, as the sensing element and an indicator ion that is similar in structure to the targeted neutral species. The sensing protocol incorporated two stages: accumulation of neutral species in the membrane phase followed by the removal of the indicator ion and potential measurement with the ISE. The sensing device was shown to be both selective and sensitive.

The potential of potentiometric sensors is becoming increasingly apparent in biomedical research with potentiometric sensors playing greater roles in pharmaceutical development, disease screening, and disease research. PVC plays a major role in membrane fabrication for ISEs showing great versatility in terms of the variety of species sensed. Kumar et al. [152] present a sensor capable of selective determination of domperidone, a clinically useful pharmaceutical for a wide variety of stomach ailments. Typically the pharmaceutical's concentration is measured using a combination of analytical techniques. The authors were able to accomplish this through the use of two separate sensors, made of a PVC membrane and a carbon plate, with the electroactive ion pair domperidonephosphotungstic.

Other groups have also reported the successful fabrication of potentiometric sensors for both drug and biological detection [153-155].

\subsubsection{Voltammetric sensors}

Voltammetry, or more specifically amperometry is a powerful, potentially highly selective analytical technique [156]. Carbon-based electrodes are widely used in voltammetric studies because of their low cost, availability, stability and processability. This has resulted in the availability of a variety of carbon-based electrodes including GC, carbon paste $(\mathrm{CP})$, polycrystalline boron doped diamond (pBDD), CNTs, and most recently graphene. Carbonbased sensors are easily modified via casting, electrodeposition, functionalisation etc. to impart selectivity. For example coupling nanostructures with high surface area with stripping voltammetry greatly enhances specificity. CNTs are ideal materials for incorporation into electrochemical sensors due to their high surface area, high aspect ratio, and enhanced catalytic properties. This is illustrated by the work of $\mathrm{Xu}$ and colleagues who developed a GC electrode modified with a nafion/bismuth/MWCNTs composite film capable of the sensitive detection $\mathrm{Pb}^{2+}$ and $\mathrm{Cd}^{2+}$ (LODs of 25 and $40 \mathrm{ppt}$ respectively) [157]. The nanocomposite allowed the sensor to exploit bismuth's wide potential window and insensitivity to $\mathrm{O}_{2}$, as well as the MWCNTs enhanced adsorptive capabilities and high surface area. The authors also noted that there was no interference exhibited by a significant number of anions, with a 500-fold mass ratio of $\mathrm{SCN}^{-}, \mathrm{Cl}^{-}, \mathrm{F}^{-}, \mathrm{PO}_{4}{ }^{3-}, \mathrm{SO}_{4}{ }^{2-}, \mathrm{NO}_{3}-$ for example showing no influence on the sensing films signal response for $\mathrm{Pb}(\mathrm{II})$ and $\mathrm{Cd}(\mathrm{II})$. 
Again much of sensor research is driven by a focus on a final application, with the demand for improved and continuous environmental monitoring driving a significant portion of sensor development. For example Zima et al report the use of numerous CPEs that incorporate nanotube or nanoparticles and are modified both chemically and biologically for the detection of various organic molecules such as pharmaceuticals and environmental pollutants. Within this work the authors describe how the performance of these sensors is enhanced, for example, a Tyrosinase modified CPE detected Cysteine with a LOD of $1-8 \mu \mathrm{M}$ with no interference from Ascorbic acid and Uric acid, similarly a CPE modified with Glucose oxidase and gold nanoparticles showed no effects in the presence these common interferents [158].

The incorporation of nanomaterials in the fabrication and development of biosensors has resulted in their improved selectivity. This is particularly evident when considering sensors for the detection of neurotransmitters such as dopamine. Shang and colleagues described the fabrication of a dopamine selective, boron-doped diamond (BDD) electrode [159]. The electrodes sensitivity (LOD of $5 \mathrm{nM}$ ) and selectivity was augmented by the anionic nature of the dopant, sulfobutylether-cyclodextrin, which pre-concentrated the dopamine and excluded common anionic interferences like ascorbic acid and uric acid. Yan et al. produced a 3,4-dihydroxyphenylacetic acid (DOPAC), a metabolite of dopamine, sensor, through the functionalising of SWCNTs that were adsorbed with cyclophane (a DOPAC recognition element) on to a GC electrode [160]. The sensor demonstrated a significant selectivity to DOPAC as the SWNT transducer coupled with a cyclophane recognition element exhibited a stronger binding affinity for DOPAC over both Ascorbic acid and Dopamine.

The incorporation of bio- materials [161, 162] into electrochemical sensors enables a level of selectivity that is comparable to that seen in nature, with the majority of advancements in biosensors due to the immobilization of a biological material (and hence it's physiochemical properties to an electrode's surface electrode [163, 164]. Mimicking nature selectivity has been attempted with the use of molecularly imprinted polymers but so far has not rivalled the selectivity of biomolecules such as antibodies, nucleic acids, aptamers, etc.

\subsection{Multi-analyte detection}

Simultaneous detection of multiple analytes is a highly desirable feature of any sensor. Current research is endeavouring to exploit the capability of voltammetric analysis to sensitively, selectively rapidly differentiate between numerous compounds. This is particularly evident in recent developments in biosensors. Recent research highlights the interest in simultaneously quantifying physiologically relevant compounds such as uric acid, ascorbic acid and/or catecholamines [165-167]. A large body of literature describes the production of a variety of sensors with multi-analyte sensing capabilities, exploiting a variety of nanomaterials including CNTs [168-170], individual nanoparticles [171] and polymer nanocomposites [172, 173] 
The electronic nose or tongue is an analytical instrument comprising an array of chemical sensors with partial specificity or cross-sensitivity to different components of a mixture/compound, and an appropriate method of pattern recognition and/or multivariate calibration for the data processing. They can quantitatively differentiate between the composition of complex liquids/gases. Such sensors have a number of applications in various industrial areas including the pharmaceutical industry and the food and beverage sector [174], where they can be used to analyse flavour ageing in beverages, quantify bitterness of dissolved compounds, quantify taste masking efficiency of formulations [175] (e.g. tablets, syrups, capsules), analyse medicines stability in terms of taste. Electronic tongue/noses are increasingly studied due to their industrial capabilities coupled with their fast and relatively cheap methodology [176]. For example, Pigani and colleagues detail their development of a poly(3,4 - ethylendioxythiophene ) modified electrode that could be incorporated into an electronic tongue. The sensor was utilised in the blind analysis of red wines, (a sample set of 144 red wines of different origin and variety) for classification and calibration purposes. Treating the data obtained from voltammetric measurements using partial least squares analysis allowed the authors to both correlate the calibration procedure results with those from traditional analytical methods and develop classification models for the wines, based on quantitative parameters and qualitative information such as the origin and variety. The sensor may also be used for quality control as it can rapid identify samples that failed to meet threshold limits for $\mathrm{SO}_{2}$, colour intensity and total polyphenols.

Similar studies by Gutierrez et al. $[177,178]$ in which the authors developed a sensing system for the classification and characterisation of both red and white wines. The sensor consisted of six ISFET sensors, a conductivity sensor, a redox potential sensor, two amperometric electrodes, a gold microelectrode and a microelectrode for sensing electrochemical oxygen demand. The system was shown to be capable of distinguishing between samples according to their grape and even geographical origin the authors also demonstrated the system's ability to differentiate between the mono - varietal samples and their mixtures further emphasise the extent of development sensing has undergone in the last decade and its expanding potential for multiple applications in a variety of industries will only encourage further development of multi-analyte sensing devices.

\subsection{Stability}

The most desirable sensors are those that retain their characteristics when tested or used under varying conditions and environments, i.e. are those which can function in a myriad of harsh conditions. Where nanomaterials are encompassed in the sensor fabrication, the resulting nanocomposites possess enhanced physical and chemical properties when compared to their constitute components depending on the chemical nature of each component and how they interact. This interaction depends strongly on characteristics of each component, i.e. interface, size, shape and structure. In extreme cases, where there is no or little interaction between the components, the composites' properties should be equivalent to a simple sum of the properties of the individual elements. In cases where the 
interaction between the constituents is strong, the properties of the composite system can differ substantially from the simple sum of the properties of the individual components. The characteristics of the individual components are lost and new feature arise as a result of the strong interaction [166].

Chik and $\mathrm{Xu}$ [179] have described a fabrication method that not only allows the addition of a variety of materials, including metals, semiconductors, and CNTs to an anodised aluminium oxide porous membrane but also, through tuning of the synthesis parameters, controls the nanomaterials morphology. This enabled the authors to engineer the physical properties of the composite by determining the shape, size, composition and doping of the nanostructures, as well as new properties produced by their interaction with the matrix itself. Some of these properties and functions were not intrinsic to the individual nano-elements but were due to the collective behaviour of the nanostructures within the membrane. The novel nanocomposite platform described potentially offers a wide range of applications in various fields including electronics, optics, mechanics, and biotechnologies.

Shi et al. [180], summarise a range of chemical sensors that apply the optical principles of nanomaterials for the detection of multiple chemical and biochemical analytes. These include colorimetric biosensors based on gold nanoparticles, gas sensing semi-conductors which exhibit changes to their optical transmittance when exposed to gases such as NO, and $\mathrm{CO}$ and optical humidity sensors based on changes in cobalt oxide films' absorbance in the visible wavelength. In this review the authors also report the development of a $\mathrm{SrCO}_{3}$ nanoparticle based ethanol sensor which displayed exceptional durability during a 100 hour reaction with $2000 \mathrm{ppm}$ ethanol at $380{ }^{\circ} \mathrm{C}$.

Chemiresistor sensors have many potential applications, including environmental monitoring [183]. However these sensors can become unstable in extreme environments. The preparation of a nanocomposite can counteract the existing weaknesses of conventional sensors by combining the strengths of nanoparticles with the composite material [184]. This is clearly illustrated in the application of nanocomposites in humidity sensing techniques. Humidity sensing materials can be grouped into two types; ceramics and polymers both possess good chemical and thermal stability, environmental adaptability and a wide range of working temperatures.

Often the sensing mechanisms these materials employ are their surface electrical conductivity or the dielectric constant, which are affected by the adsorption of water vapour. Polymer-based humidity sensing materials possess some advantages in comparison to ceramics; including a higher sensitivity, decreased humidity hysteresis, low cost, flexibility and easy processability [180]. For example, Wang et al. [185] describe the improved humidity sensing capability and stability seen by combining nano $\mathrm{BaTiO}_{3}$ with acrylic resin as a nanocomposite humidity sensor. The authors determined that the electrical properties of the sensor, including resistance versus relative humidity, humidity hysteresis, response recovery time and long term stability of the composite sensor were better than that of a sensor composed just of the nano $\mathrm{BaTiO}_{3}$. Similar research has led to multiple reports on 
chemical polymerisation and electrochemical techniques for the preparation of polymer nanocomposites for gas and humidity sensor applications [186-188].

Similarly the stability of biosensor may be significantly enhanced through the incorporation of nanomaterials [189, 190]. For example, Gopalan et al. fabricated an electrochemical biosensor-based on a multi-component composite comprising of MWNT, silica, Nafion and PANI. This blend of these materials within the sensing composite resulted in excellent electrocatalytic activity, sensitivity and stability. Silica gave the composite mechanical stability due to it's rigid 3D porous structure and the inclusion of the MWCNTs was also benefited the sensors mechanical rigidity. Moreover the incorporation of Nafion minimised the brittleness of the pure sol-gel derived silica and enhanced the sensor's long-term stability. The authors utilised this organic-inorganic nanocomposite as a glucose biosensor possessing enhanced stability over time. The sensor displayed a reproducible current response (R.S.D. $<2.2 \%$ ) for 10 measurements. The stability of the Nafion-silica/MWNT-gPANI/GOx biosensor was determined by storing the biosensor at $4{ }^{\circ} \mathrm{C}$ in PBS (pH 7) for 20 days and monitoring the response current daily for $1.0 \mathrm{mM}$ of glucose at $+0.20 \mathrm{~V}$. The biosensor retained its $93 \%$ current response after 20 days by loading GOx within the nanocomposite [191].

Similarly Guell and co-workers [192] detail a study of the differing characteristics of three carbon-based electrodes: GC, pBDD, and CNTs. The authors determined that "pristine" CNT networks exhibited background current densities that were 2 orders of magnitude lower than GC and 20 times lower than pBDD for the detection of serotonin, however pBDD electrodes underwent significantly less fouling (minimised by optimisation of the potential range) than the CNTs electrode and exhibited superior stability which is often attributed to the H-surface termination of as - grown pBDD electrodes.

Apetrei et al. compared CPEs prepared with graphite, carbon microspheres, or MWCNTs. The electrodes made using carbon microspheres were the most stable (giving consistent results for successive measurement of catechol, with a relative standard deviation of just $3.2 \%$ ) and gave the best performances in terms of kinetics. Their work also demonstrated that an array combining the three electrode types could discriminate between a number of anti-oxidants depending on their chemical structure and reactivity [193].

Strategies for enhancing the stability of electrochemical sensors continue as advances already made result in the application of sensors in a variety of environments previous considered excessively harsh, e.g. extreme temperatures [194], both high and low pH [195]. Analytical chemists have overcome such challenges by both incorporating biomolecules into sensor platforms and exploiting the desirable properties of novel materials with nanotechnology allowing the development of a variety of disposable and long-life sensors.

\section{In summary}

Electrochemical sensors have a long and rich history in the field of analytical chemistry contributing to multiple industries. The impact of electrochemical sensors is clearly 
illustrated by their multiple every day applications and the sheer number of commercially available sensors available. This is evident especially in the field of potentiometric sensors where the further development of ISEs remains the mainstay of recent research. The enhancement of such devices sensing abilities in terms of sensitivity, selectivity, and stability through the incorporation of both novel ionophores into sensor membranes and strategies including analyte preconcentration and background subtraction has allowed the effective detection of cations, anions, and neutral species in challenging environments (conditions with high concentrations of background analytes and biological milieu).

As the demand for sensitive, rapid, and selective determination of analytes continues to grow, so too does the utility of electrochemical sensors. Unlike their spectroscopic and chromatographic counterparts, they are readily adapted for the detection of a wide range of analytes and may be incorporated into robust, portable and/or miniaturised devices while remaining relatively inexpensive.

Despite their ubiquitous presence, electrochemical sensors remain a dynamic field of research especially when coupled with the continued expansion of nanoscience and nanotechnology. Nanomaterials already have extensive applications in electrochemical sensors systems with a significant potential for future development. This is primarily due to the unique and attractive properties that nanoparticles and nanostructures exhibit, the exploitation of which allows the development of electroanalytical systems exhibiting similarly attractive analytical behaviours. A wide variety of metallic and organic nanomaterials have been used to fabricate a diverse range of electrochemical sensing systems, based on their special physical, chemical and even biological properties. Further development of electroanalytical sensor technology, with the discovery and subsequent exploitation of novel properties of nanomaterials will only result in the further evolution of electrochemical sensing platforms.

The majority of modern electrochemical sensor development focuses on the incorporation of both microfabrication and nanofabrication to design sensors of a smaller size and hence lower power demands, of lower cost, and improved portability. The miniaturisation of electrodes continuing down to the nano regime has allowed measurements of fast electron kinetics at very low analyte concentrations. The demand for increasingly low detection levels has been met with the production of arrays. The incorporation of nanostructures has augmented the electrode reactive surface area even with less material used in the physical electrode allowing greater signal to noise ratio for a given analyte.

The development of sensors for the measurement of neurotransmitters is a major trend emerging in voltammetric sensor research, with the sensitivity and selectivity of such sensors being greatly improved due to the development of both measurement techniques and electrode materials. A recent shift in focus from glucose analysis to other physiologically analytes, has driven biosensor development, with DNA hybridization and immunological recognition being the basis of a significant portion of new electrochemical detectors. Again the application of nanomaterials has enabled significant improvements in the analytical performance and utility of biosensors. 
In conclusion, the field of electrochemical sensors continues to grow and develop new applications at pace. The incorporation and interaction of unique materials, both nano and biological, remains the focus of a significant portion of research. This will likely continue as the production of sensors with increased specificity and sensors capable of simultaneous determinations with the ability to operate in complex matrixes remains the long term focus of sensor research.

\section{Abbreviations}

2-diisopropylaminoethanethiol

Alternating Current

DIPAET

Boron - Doped Diamond

AC

Carbon Nanotube

BDD

Carbon Paste Electrode

CNT

Coated Wire Electrodes

CPE

Deoxyribonucleic Acid

CWES

Electrochemical Impedance Spectroscopy

DNA

Ethylenediaminetetraacetic acid

EIS

Femtograms

EDTA

Glassy Carbon

fg

Glutathione

GC

Hepatitis B surface Antigen

GSH

Human Serum Albumin

HBsA

Indium Tin Oxide

HSA

Ion Selective Electrode

ITO

Light Addressable Potentiometric Sensors

ISE

Localised Surface Plasmon Resonance

LAPS

Methyldopa

LSPR

Millilitres

MDA

Molecularly Imprinted Polymers

$\mathrm{ml}$

Nanograms

National Nanotechnology Initiative

MIPs

Nicotinamide Adenine Dinucleotide

ng

Parts per Billion

NNI

Persistent Organic Pollutants

$\mathrm{NADH}$

Poly(3,4-ethylenedioxythiophene)

Poly(styrene-co-acrylic Acid)

$\mathrm{ppb}$

POPs

PEDOT

Polyaniline

PSA

Poly-Dopamine

PANI

Prussian Blue

PDOP

Quantum Dots

$\mathrm{PB}$

Room Temperature Ionic Liquids

QDs

Single Walled Carbon Nanotube

RTIL

Standard Hydrogen Electrode 
Three Dimensional

3D

3,4-dihydroxyphenylacetic acid

DOPAC

Anodic stripping voltammetry

ASV

Carcinoembryonic Antigen

CEA

Carbon Nanotube Epoxy Composite Electrodes

CNTECE

Chemically Modified Graphene

CMG

Cyclic Voltammetry

$\mathrm{CV}$

Dichlorodiphenyltrichloroethane

DDT

Electrospun Carbon Nanofiber - Modified Carbon Paste Electrode

ECF-CPE

Federation of European Chemical Societies

FECS

Field Effect Transistor

FET

Glucose Oxidase

GOD

Graphene Nanoribbon

GNR

Hexanedithiol

HDT

Immunoglobulin G

International Union of Pure and Applied Chemistry

$\operatorname{IgG}$

Ion Selective Field Effect Transistor

IUPAC

ISFET

Limit of Detection

LOD

Mercapto-1Hexanol

$\mathrm{MCH}$

Microcystin-LR

MC-LR

milli-molar

$\mathrm{mM}$

Multi Walled Carbon Nanotube

MWCNT

Nanoparticles

NPs

Nitrate Reductase

NR

Nuclear Fast Red

NFR

ppm

PoC

Point of Care

PEDOT:PSS

Poly(3,4-ethylenedioxythiophene) : Poly(styrenesulfonic Acid)

PSS

Polycrystalline Boron Doped Diamond

pBDD

Polyvinyl chloride

PVC

Prussian White

PW

Relative Humidity

$\mathrm{RH}$

Screen Printed Electrode

SPE

Specific Thiolated Capture Probe

SHCP

Surface Plasmon Resonance

SPR

Trinitrotoluene

TNT

\section{Author details}

Aoife C. Power and Aoife Morrin

National Centre of Sensor Research,

School of Chemical Sciences, Dublin City University, Glasnevin, Dublin, Ireland 


\section{References}

[1] Niinisto L (Chairperson), Working party on Analytical Chemistry (WPAC). In Federation of European Chemical Societies (FECS). 1993. Edingburgh

[2] Cremer M, (1906), Über die Ursache der elektromotorischen Eigenschaften der Gewebe, zugleich ein Beitrag zur Lehre von den polyphasischen Elektrolytketten, Zeitschrift fur Biologie, 47: 562 - 608.

[3] Haber F and Klemensiewicz Z, (1909), Über elektrische Phasengrenzkräfte Zeitschrift für Physikalische Chemie, 67: 385 - 431.

[4] Lubert KH and Kalcher K, (2010), History of Electroanalytical Methods, Electroanalysis, 22: 1937-1946.

[5] Anker JN, et al., (2008), Biosensing with plasmonic nanosensors, Nature Materials, 7: 442-453.

[6] Yi F and La Van DA, (2012), Nanoscale Thermal Analysis for Nanomedicine by Nanocalorimetry, Wiley Interdisciplinary Reviews - Nanomedicine and Nanobiotechnology, 4: 31-41.

[7] Waggoner PS and Craighead HG, (2007), Micro- and Nanomechanical Sensors for Environmental, Chemical and Biological Detection, Lab on a Chip, 7: 1238-1255.

[8] Holford TRJ, Davis F, and Higson SPJ, (2012), Recent trends in antibody based sensors, Biosensors \& Bioelectronics, 34: 12-24.

[9] Palchetti I and Mascini M, (2012), Electrochemical Nanomaterial - Based Nucleic Acid Aptasensors, Analytical and Bioanalytical Chemistry, 402: 3103-3114.

[10] Perfezou M, Turner A, and Merkoci A, (2012), Cancer Detection Using Nanoparticle Based Sensors, Chemical Society Reviews, 41: 2606-2622.

[11] Lei J and Ju H, (2012), Signal Amplification Using Functional Nanomaterials for Biosensing, Chemical Society Reviews, 41: 2122-2134.

[12] Liu Y, Dong X, and Chen P, (2012), Biological and Chemical Sensors Based on Graphene Materials, Chemical Society Reviews, 41: 2283-2307.

[13] Sun YF, et al., (2012), Metal Oxide Nanostructures and Their Gas Sensing Properties: A Review, Sensors, 12: 2610-2631.

[14] Silvester DS, (2011), Recent Advances in the use of Ionic Liquids for Electrochemical Sensing, Analyst, 136: 4871-4882.

[15] Ni YN and Kokot S, (2008), Does Chemometrics Enhance the Performance of Electroanalysis?, Analytica Chimica Acta, 626: 130-146.

[16] Adibi M, Pirali-Hamedani M, and Norouzi P, (2011), Copper Nano-composite Potentiometric Sensor, International Journal of Electrochemical Science, 6: 717-726.

[17] Lin P and Yan F, (2012), Organic Thin-Film Transistors for Chemical and Biological Sensing, Advanced Materials, 24: 34-51.

[18] Scampicchio M, et al., (2012), Electrospun Nonwoven Nanofibrous Membranes for Sensors and Biosensors, Electroanalysis, 24: 719-725.

[19] Kimmel DW, LeBlanc G, Meschievitz ME, and Cliffel D, (2012), Electrochemical Sensors and Biosensors, Analytical Chemistry, 84: 685-707. 
[20] Chen W, et al., (2012), Recent advances in electrochemical sensing for hydrogen peroxide: a review, Analyst, 137: 49-58.

[21] Shan X, et al., (2010), Imaging Local Electrochemical Current via Surface Plasmon Resonance, Science, 327: 1363-1366.

[22] (2007), Electrochemical Sensor Analysis, Elsevier:

[23] Zhang XJ, Ju H, and Wang J, (2007), Electrochemical Sensors, Biosensors and their Biomedical Application, Elsevier:

[24] Janata J, (2009), Principles of chemical sensors, Springer Verlag, Dorfrecht, Heidelberg, London, New York:

[25] Bobacka J, Ivaska A, and Lewenstam A, (2008), Potentiometric ion sensors, Chemical Reviews, 108: 329-351.

[26] Bratov A, Abramova N, and Ipatov A, (2010), Recent trends in potentiometric sensor arrays - A review, Analytica Chimica Acta, 678: 149-159.

[27] Faridbod F, Ganjali MR, Dinarvand R, and Norouzi P, (2008), Developments in the field of conducting and non-conducting polymer based potentiometric membrane sensors for ions over the past decade, Sensors, 8: 2331-2412.

[28] Xu L, et al., (2012), An Enantioselective Polyaniline - Coated Membrane Electrode Based on Chiral Salen Mn(III) as Chiral Selector, Analytical Methods, 4: 807-811.

[29] Evtugyn G A, et al., (2008), Selectivity of solid-contact Ag potentiometric sensors based on thiacalix 4 arene derivatives, Talanta, 76: 441-447.

[30] Mattinen U, Rabiej S, Lewenstam A, and Bobacka J, (2011), Impedance Study of the Ion to - Electron Transduction Process for Carbon Cloth as Solid - Contact Material in Potentiometric Ion Sensors, Electrochimica Acta, 56: 10683-10687.

[31] Rius-Ruiz FX, et al., (2011), Potentiometric Strip Cell Based on Carbon Nanotubes as Transducer Layer: Toward Low - Cost Decentralized Measurements, Analytical Chemistry, 83: 8810-8815.

[32] Pesavento M, et al., (2012), Ion Selective Electrode for Dopamine Based on a Molecularly Imprinted Polymer, Electroanalysis, 24: 813-824.

[33] Li F, et al., (2012), All - Solid - State Potassium - Selective Electrode Using Graphene as the Solid Contact, Analyst, 137: 618-623.

[34] Jia Y, et al., (2011), Bio-Initiated Light Addressable Potentiometric Sensor for Unlabeled Biodetection and its MEDICI Simulation, Analyst, 136: 4533-4538.

[35] Liu Q, et al., (2011), In vitro Assessing the Risk of Drug - Induced Cardiotoxicity by Embryonic Stem Cell - Based Biosensor, Sensors and Actuators B-Chemical, 155: 214219.

[36] Gupta VK, et al., (2011), Voltammetric techniques for the assay of pharmaceuticals - A review, Analytical Biochemistry, 408: 179-196.

[37] Granado Rico MA, Olivares-Marin M, and Pinilla Gil E, (2009), Modification of carbon screen-printed electrodes by adsorption of chemically synthesized Bi nanoparticles for the voltammetric stripping detection of $\mathrm{Zn}(\mathrm{II}), \mathrm{Cd}(\mathrm{II})$ and $\mathrm{Pb}(\mathrm{II})$, Talanta, 80: 631-635. 
[38] Fu XC, et al., (2011), Stripping voltammetric detection of mercury(II) based on a surface ion imprinting strategy in electropolymerized microporous poly(2mercaptobenzothiazole) films modified glassy carbon electrode, Analytica Chimica Acta, 685: 21-28.

[39] Mohadesi A, Teimoori E, Taher MA, and Beitollah H, (2011), Adsorptive Stripping Voltammetric Determination of Cobalt (II) on the Carbon Paste Electrode, International Journal of Electrochemical Science, 6: 301-308.

[40] Somerset V, et al., (2010), Development and Application of a Poly(2,2 '- Dithiodianiline) (PDTDA) - Coated Screen - Printed Carbon Electrode in Inorganic Mercury Determination, Electrochimica Acta, 55: 4240-4246.

[41] Fojta M, Jelen F, Havran L, and Palecek E, (2008), Electrochemical stripping techniques in analysis of nucleic acids and their constituents, Current Analytical Chemistry, 4: 250262.

[42] Clark LC, (1956), Monitor and Control of Blood and Tissue Oxygen Tensions, Transactions American Society for Artificial Internal Organs, 2: 41-46.

[43] Clark LC and Lyons C, (1962), Electrode Systems for Continuous Monitoring in Cardiovascular Surgery, Annals of the New York Academy of Sciences, 102: 29-45.

[44] Hu CG, et al., (2012), Inkjet Printing of Nanoporous Gold Electrode Arrays on Cellulose Membranes for High-Sensitive Paper-Like Electrochemical Oxygen Sensors Using Ionic Liquid Electrolytes, Analytical Chemistry, 84: 3745-3750.

[45] Morrin A, (2012), Inkjet Printed Electrochemical Sensors, In Korvink JG, et al.s Inkjetbased Micromanufacturing, Wiley-VCH: 295-309.

[46] Newman JD and Turner APF, (2005), Home Blood Glucose Biosensors: A Commercial Perspective, Biosensors \& Bioelectronics, 20: 2435-2453.

[47] Zhong HA, et al., (2012), Non - Enzymatic Hydrogen Peroxide Amperometric Sensor Based on a Glassy Carbon Electrode Modified with an MWCNT / Polyaniline Composite Film and Platinum Nanoparticles, Microchimica Acta, 176: 389-395.

[48] Guo YJ, Guo SJ, Fang YX, and Dong SJ, (2010), Gold nanoparticle/carbon nanotube hybrids as an enhanced material for sensitive amperometric determination of tryptophan, Electrochimica Acta, 55: 3927-3931.

[49] Ventura DN, et al., (2012), A Flexible Cross - Linked Multi - Walled Carbon Nanotube Paper for Sensing Hydrogen, Carbon, 50: 2672-2674.

[50] Kang E, et al., (2012), Electrospun BMIMPF6/Nylon 6,6 Nanofiber Chemiresistors as Organic Vapour Sensors, Macromolecular Research, 20: 372-378.

[51] Crowley K, et al., (2008), Fabrication of an ammonia gas sensor using inkjet-printed polyaniline nanoparticles, Talanta, 77: 710-717.

[52] Rheaume JM and Pisano AP, (2011), A Review of Recent Progress in Sensing of Gas Concentration by Impedance Change, Ionics, 17: 99-108.

[53] Bataillard P, et al., (1988), Direct Detection of Immunospecies by Capacitance Measurements, Analytical Chemistry, 60: 2374-2379. 
[54] Tully E, Higson SP, and Kennedy RO, (2008), The Development of a 'Labeless' Immunosensor for the Detection of Listeria Monocytogenes Cell Surface Protein, Internalin B, Biosensors \& Bioelectronics, 23: 906-912.

[55] Caballero D, et al., (2012), Impedimetric immunosensor for human serum albumin detection on a direct aldehyde-functionalized silicon nitride surface, Analytica Chimica Acta, 720: 43-48.

[56] Loo AH, et al., (2012), Impedimetric Immunoglobulin G Immunosensor Based on Chemically Modified Graphenes, Nanoscale, 4: 921-925.

[57] Yang M, et al., (2011), Site - Specific Immobilization of Gold Binding Polypeptide on Gold Nanoparticle - Coated Graphene Sheet for Biosensor Application, Nanoscale, 3: 2950-2956.

[58] Ahmadalinezhad A., Wu G. S., and Chen A. C., (2011), Mediator-free electrochemical biosensor based on buckypaper with enhanced stability and sensitivity for glucose detection, Biosensors \& Bioelectronics, 30: 287-293.

[59] Yehezkeli O, Tel-Vered R, Reichlin S, and Willner I, (2011), Nano - Engineered Flavin Dependent Glucose Dehydrogenase / Gold Nanoparticle - Modified Electrodes for Glucose Sensing and Biofuel Cell Applications, ACS Nano, 5: 2385-2391.

[60] Wipawakarn P, Ju HX, and Wong DKY, (2012), A Label - Free Electrochemical DNA Biosensor Based on a $\mathrm{Zr}(\mathrm{IV})$ - Coordinated DNA Duplex Immobilised on a Carbon Nanofibre Chitosan Layer, Analytical and Bioanalytical Chemistry, 402: 2817-2826.

[61] Lahiff E, et al., (2010), The Increasing Importance of Carbon Nanotubes and Nanostructured Conducting Polymers in Biosensors, Analytical and Bioanalytical Chemistry, 398: 1575-1589.

[62] Ansari S. A. and Husain Q., (2012), Potential applications of enzymes immobilized on/in nano materials: A review, Biotechnology Advances, 30: 512-523.

[63] Xiao Y, et al., (2003), "Plugging into Enzymes": Nanowiring of Redox Enzymes by a Gold Nanoparticle, Science, 299: 1877-1881.

[64] Zayats M, Willner B, and Willner I, (2008), Design of Amperometric Biosensors and Biofuel Cells by the Reconstitution of Electrically Contacted Enzyme Electrodes, Electroanalysis, 20: 583-601.

[65] Sia SK and Chin CD, (2011), Analytical Chemistry: Sweet Solution to Sensing, Nature Chemistry, 3: 659-660.

[66] Xiang Y and Lu Y, (2011), Using Personal Glucose Meters and Functional DNA Sensors to Quantify a Variety of Analytical Targets, Nature Chemistry, 3: 697-703.

[67] Lai GS, et al., (2012), Electrochemical Stripping Analysis of Nanogold Label - Induced Silver Deposition for Ultrasensitive Multiplexed Detection of Tumor Markers, Analytica Chimica Acta, 721: 1-6.

[68] Joung CK, et al., (2012), Ultra-Sensitive Detection of Pathogenic Microorganism Using Surface - Engineered Impedimetric Immunosensor, Sensors and Actuators B-Chemical, 161: 824-831. 
[69] Cataldo V, Vaze A, and Rusling JF, (2008), Improved detection limit and stability of amperometric carbon nanotube-based immunosensors by crosslinking antibodies with polylysine, Electroanalysis, 20: 115-122.

[70] Lin DJ, et al., (2012), Triple Signal Amplification of Graphene Film, Polybead Carried Gold Nanoparticles as Tracing Tag and Silver Deposition for Ultrasensitive Electrochemical Immunosensing, Analytical Chemistry, 84: 3662-3668.

[71] Peng J, et al., (2012), Calcium Carbonate - Gold Nanocluster Hybrid Spheres: Synthesis and Versatile Application in Immunoassays, Chemistry - a European Journal, 18: 52615268.

[72] Zhang DW, et al., (2012), A Label - Free Aptasensor for the Sensitive and Specific Detection of Cocaine Using Supramolecular Aptamer fragments / Target Complex by Electrochemical Impedance Spectroscopy, Talanta, 92: 65-71.

[73] Ohno Y, Maehashi K, and Matsumoto K, (2010), Label - Free Biosensors Based on Aptamer - Modified Graphene Field - Effect Transistors, Journal of the American Chemical Society, 132: 18012-18013.

[74] Renedo OD, Alonso-Lomillo MA, and Martinez MJ, (2007), Recent Developments in the Field of Screen - Printed Electrodes and their Related Applications, Talanta, 73: 202-219.

[75] Gonzalez-Macia L, Smyth MR, and Killard AJ, (2012), A Printed Electrocatalyst for Hydrogen Peroxide Reduction, Electroanalysis, 24: 609-614.

[76] Hu JY, Lin YP, and Liao YC, (2012), Inkjet Printed Prussian Blue Films for Hydrogen Peroxide Detection, Analytical Sciences, 28: 135-140.

[77] Liu H and Crooks RM, (2012), Paper - Based Electrochemical Sensing Platform with Integral Battery and Electrochromic Read-Out, Analytical Chemistry, 84: 2528-2532.

[78] Anastasova-Ivanova S, et al., (2010), Development of miniature all-solid-state potentiometric sensing system, Sensors and Actuators B: Chemical, 146: 199-205.

[79] Mousavi Z, et al., (2011), Comparison of Multi - Walled Carbon Nanotubes and Poly (3,octylthiophene) as Ion - to - Electron Transducers in All - Solid - State Potassium Ion Selective Electrodes, Electroanalysis, 23: 1352-1358.

[80] Li C, Bai H, and Shi G, (2009), Conducting Polymer Nanomaterials: Electrosynthesis and Applications, Chemical Society Reviews, 38: 2397-2409.

[81] Dahlin AB, et al., (2012), Electrochemical plasmonic sensors, Analytical and Bioanalytical Chemistry, 402: 1773-1784.

[82] Makowski MS and Ivanisevic A, (2011), Molecular Analysis of Blood with Micro- / Nanoscale Field Effect Transistor Biosensors, Small, 7: 1863-1875.

[83] Wang J, (2008), Electrochemical Glucose Biosensors, Chemical Reviews, 108: 814-825.

[84] Kagie A, et al., (2008), Flexible Rolled Thick Film Miniaturized Flow Cell for Minimally Invasive Amperometric Sensing, Electroanalysis, 20: 1610-1614.

[85] Metters JP, Kadara RO, and Banks CE, (2011), New Directions in Screen Printed Electroanalytical Sensors: An Overview of Recent Developments, Analyst, 136: 10671076 . 
[86] Kuralay F, Campuzano S, Haake DA, and Wang J, (2011), Highly Sensitive Disposable Nucleic Acid Biosensors for Direct Bioelectronic Detection in Raw Biological Samples, Talanta, 85: 1330-1337.

[87] Malzahn K, et al., (2011), Wearable Electrochemical Sensors for in situ Analysis in Marine Environments, Analyst, 136: 2912-2917.

[88] Sriprachuabwong C, et al., (2012), Inkjet - Printed Graphene - PEDOT: PSS Modified Screen Printed Carbon Electrode for Biochemical Sensing, Journal of Materials Chemistry, 22: 5478-5485.

[89] Kadara RO, Jenkinson N, and Banks CE, (2009), Screen printed recessed microelectrode arrays, Sensors and Actuators B: Chemical, 142: 342-346.

[90] Amatore C, Oleinick A, and Svir I, (2008), Theoretical Analysis of Microscopic Ohmic Drop Effects on Steady-State and Transient Voltammetry at the Disk Microelectrode: A Quasi-Conformal Mapping Modeling and Simulation, Analytical chemistry, 80: 79477956.

[91] Amatore C, Oleinick AI, and Svir I, (2009), Numerical Simulation of Diffusion Processes at Recessed Disk Microelectrode Arrays Using the Quasi-Conformal Mapping Approach, Analytical chemistry, 81: 4397-4405.

[92] Guo J and Lindner E, (2008), Cyclic voltammograms at coplanar and shallow recessed microdisk electrode arrays: Guidelines for design and experiment, Analytical chemistry, 81: 130-138.

[93] Li Y, Bergman D, and Zhang B, (2009), Preparation and Electrochemical Response of 13 nm Pt Disk Electrodes, Analytical chemistry, 81: 5496-5502.

[94] Dumitrescu I, Unwin PR, Wilson NR, and Macpherson JV, (2008), Single-Walled Carbon Nanotube Network Ultramicroelectrodes, Analytical chemistry, 80: 3598-3605.

[95] Hallam PM, Kampouris DK, Kadara RO, and Banks CE, (2010), Graphite screen printed electrodes for the electrochemical sensing of chromium (VI), Analyst, 135: 1947-1952.

[96] Khairy M, Kadara RO, Kampouris DK, and Banks CE, (2010), In Situ Bismuth Film Modified Screen Printed Electrodes for the Bio - Monitoring of Cadmium in Oral (Saliva) Fluid, Analytical Methods, 2: 645-649.

[97] Liu C, Hayashi K, and Toko K, (2011), Au Nanoparticles Decorated Polyaniline Nanofiber Sensor for Detecting Volatile Sulfur Compounds in Expired Breath, Sensors and Actuators B: Chemical, 161: 504-509.

[98] Toda K, Li J, and Dasgupta PK, (2006), Measurement of Ammonia in Human Breath with a Liquid - Film Conductivity Sensor, Analytical chemistry, 78: 7284-7291.

[99] Van den Velde S, Nevens F, van Steenberghe D, and Quirynen M, (2008), GC - MS Analysis of Breath Odor Compounds in Liver Patients, Journal of Chromatography B, 875: 344-348.

[100] Hibbard T and Killard AJ, (2011), Breath ammonia analysis: Clinical application and measurement, Critical Reviews in Analytical Chemistry, 41: 21-35.

[101] Power AC, Betts AJ, and Cassidy JF, (2010), Silver Nanoparticle Polymer Composite Based Humidity Sensor, Analyst, 135: 1645-1652. 
[102] Pichetsumthorn P, Vattipalli K, and Prasad S, (2012), Nanoporous Impedemetric Biosensor for Detection of Trace Atrazine from Water Samples, Biosensors \& Bioelectronics, 32: 155-162.

[103] Neves MMPS, Gonzalez-Garcia MB, Santos-Silva A, and Costa-Garcia A, (2012), Voltammetric Immunosensor for the Diagnosis of Celiac Disease Based on the Quantification of Anti - Gliadin Antibodies, Sensors and Actuators B-Chemical, 163: 253-259.

[104] Rosales-Rivera LC, et al., (2012), Amperometric Immunosensor for the Determination of IgA Deficiency in Human Serum Samples, Biosensors \& Bioelectronics, 33: 134-138.

[105] Yu X, Kim SN, Papadimitrakopoulos F, and Rusling JF, (2005), Protein Immunosensor Using Single - Wall Carbon Nanotube Forests with Electrochemical Detection of Enzyme Labels, Molecular Biosystems, 1: 70-78.

[106] Hong CL, et al., (2012), A strategy for signal amplification using an amperometric enzyme immunosensor based on HRP modified platinum nanoparticles, Journal of Electroanalytical Chemistry, 664: 20-25.

[107] Su HL, Yuan R, Chai YQ, and Zhuo Y, (2012), Enzyme-nanoparticle conjugates at oilwater interface for amplification of electrochemical immunosensing, Biosensors \& Bioelectronics, 33: 288-292.

[108] Wang GF, et al., (2012), A Supersandwich Multienzyme - DNA Label Based Electrochemical Immunosensor, Chemical Communications, 48: 720-722.

[109] Wei Q et al., (2011), Nanoporous PtRu Alloy Enhanced Nonenzymatic Immunosensor for Ultrasensitive Detection of Microcystin - LR, Advanced Functional Materials, 21: 4193-4198.

[110] Dequaire M, Degrand C, and Limoges B, (2000), An electrochemical metalloimmunoassay based on a colloidal gold label, Analytical chemistry, 72: 55215528.

[111] Authier L, Grossiord C, Brossier P, and Limoges B, (2001), Gold nanoparticle-based quantitative electrochemical detection of amplified human cytomegalovirus DNA using disposable microband electrodes, Analytical Chemistry, 73: 4450-4456.

[112] Shen GY and Zhang Y, (2010), Highly Sensitive Electrochemical Stripping Detection of Hepatitis B Surface Antigen Based on Copper - Enhanced Gold Nanoparticle Tags and Magnetic Nanoparticles, Analytica Chimica Acta, 674: 27-31.

[113] Sakai G, Matsunaga N, Shimanoe K, and Yamazoe N, (2001), Theory of Gas - Diffusion Controlled Sensitivity for Thin Film Semiconductor Gas Sensor, Sensors and Actuators B-Chemical, 80: 125-131.

[114] Xu CN, Tamaki J, Miura N, and Yamazoe N, (1991), Grain-Size Effects on Gas Sensitivity of Porous $\mathrm{SNO}_{2}$-Based Elements, Sensors and Actuators B-Chemical, 3: 147155.

[115] Belle CJ, et al., (2011), Size dependent gas sensing properties of spinel iron oxide nanoparticles, Sensors and Actuators B-Chemical, 160: 942-950. 
[116] Meng FL, et al., (2010), Nanocomposites of Sub-10 nm $\mathrm{SnO}_{2}$ Nanoparticles and MWCNTs for Detection of Aldrin and DDT, Analytical Methods, 2: 1710-1714.

[117] Shipway AN, Katz E, and Willner I, (2000), Nanoparticle Arrays on Surfaces for Electronic, Optical and Sensor Applications, ChemPhysChem, 1: 18-52.

[118] Katz E, Willner I, and Wang J, (2004), Electroanalytical and Bioelectroanalytical Systems Based on Metal and Semiconductor Nanoparticles, Electroanalysis, 16: 19-44.

[119] Bakker E and Pretsch E, (2008), Nanoscale Potentiometry, TrAC Trends in Analytical Chemistry, 27: 612-618.

[120] Bobacka J and Ivaska A, (2010), Chemical Sensors Based on Conducting Polymers, Electropolymerization: 173-187.

[121] Long YZ, et al., (2011), Recent Advances in Synthesis, Physical Properties and Applications of Conducting Polymer Nanotubes and Nanofibers, Progress in Polymer Science, 36: 1415-1442.

[122] Lange U and Mirsky VM, (2011), Chemiresistors Based on Conducting Polymers: A Review on Measurement Techniques, Analytica Chimica Acta, 687: 105-113.

[123] Xia L, Wei Z, and Wan M, (2010), Conducting Polymer Nanostructures and their Application in Biosensors, Journal of Colloid and Interface Science, 341: 1-11.

[124] Faridbod F, Norouzi P, Dinarvand R, and Ganjali MR, (2008), Developments in the field of conducting and non-conducting polymer based potentiometric membrane sensors for ions over the past decade, Sensors, 8: 2331-2412.

[125] On JH, et al., (2009), Synthesis of 7- Deoxycholic Amides or Cholanes Containing Distinctive Ion - Recognizing Groups at C3 and C12 and Evaluation for Ion - Selective Ionophores, Tetrahedron, 65: 1415-1423.

[126] Mashhadizadeh MH, Shockravi A, Khoubi Z, and Heidarian D, (2009), Efficient Synthesis of a New Podand and Application as a Suitable Carrier for Silver Ion Selective Electrode, Electroanalysis, 21: 1041-1047.

[127] Li XG, Ma XL, and Huang MR, (2009), Lead (II) Ion - Selective Electrode Based on Polyaminoanthraquinone Particles with Intrinsic Conductivity, Talanta, 78: 498-505.

[128] Gupta VK, et al., (2008), Electroanalytical studies on cobalt (II) selective potentiometric sensor based on bridge modified calixarene in poly (vinyl chloride), Electrochimica Acta, 53: 5409-5414.

[129] Boswell PG, et al., (2005), Coordinative properties of highly fluorinated solvents with amino and ether groups, Journal of the American Chemical Society, 127: 16976-16984.

[130] Boswell PG, et al., (2008), Fluorophilic Ionophores for Potentiometric pH Determinations with Fluorous Membranes of Exceptional Selectivity, Analytical chemistry, 80: 2084-2090.

[131] Lai CZ, et al., (2009), Fluorous polymeric membranes for ionophore-based ion-selective potentiometry: how inert is Teflon AF?, Journal of the American Chemical Society, 131: 1598-1606. 
[132] Crespo GA, Gugsa D, Macho S, and Rius FX, (2009), Solid-contact pH-selective electrode using multi-walled carbon nanotubes, Analytical and Bioanalytical Chemistry, 395: 2371-2376.

[133] Crespo GA, Macho S, and Rius FX, (2008), Ion-selective electrodes using carbon nanotubes as ion-to-electron transducers, Analytical chemistry, 80: 1316-1322.

[134] Crespo GA, Macho S, Bobacka J, and Rius FX, (2008), Transduction mechanism of carbon nanotubes in solid-contact ion-selective electrodes, Analytical chemistry, 81: 676681.

[135] Parra EJ, et al., (2009), Ion - Selective Electrodes Using Multi - Walled Carbon Nanotubes as Ion - to - Electron Transducers for the Detection of Perchlorate, Analyst, 134: 1905-1910.

[136] Faridbod F, et al., (2010), $\mathrm{Ho}^{3+}$ carbon paste sensor based on multi-walled carbon nanotubes: Applied for determination of holmium content in biological and environmental samples, Materials Science and Engineering: C, 30: 555-560.

[137] Ganjali MR, et al., (2010), Determination of $\mathrm{Pb}^{2+}$ ions by a modified carbon paste electrode based on multi-walled carbon nanotubes (MWCNTs) and nanosilica, Journal of hazardous materials, 173: 415-419.

[138] Norouzi P, et al., (2010), ER ${ }^{3+}$ Carbon Paste Electrode Based on New Nano-Composite, International Journal of Electrochemical Science, 5: 367-376.

[139] Mashhadizadeh MH, Khani H, and Shockravi A, (2010), Used a New Aza - Thia Macrocycle as a Suitable Carrier in Potentiometric Sensor of Copper (II), Journal of Inclusion Phenomena and Macrocyclic Chemistry, 68: 219-227.

[140] Petković BB, et al., (2010), A Copper (II) Ion-Selective Potentiometric Sensor Based on $\mathrm{N}, \mathrm{N}^{\prime}, \mathrm{N}$ ", N"' Tetrakis (2,pyridylmethyl) 1, 4, 8, 11, Tetraazacyclotetradecane in PVC Matrix, Electroanalysis, 22: 1894-1900.

[141] Abbaspour A, Mirahmadi E, Khalafi-Nejad A, and Babamohammadi S, (2010), A highly selective and sensitive disposable carbon composite PVC-based membrane for determination of lead ion in environmental samples, Journal of hazardous materials, 174: 656-661.

[142] Hosseini M, Abkenar SD, Ganjali MR, and Faridbod F, (2011), Determination of zinc (II) ions in waste water samples by a novel zinc sensor based on a new synthesized Schiff's base, Materials Science and Engineering: C, 31: 428-433.

[143] Motlagh MG, Taher MA, and Ahmadi A, (2010), PVC membrane and coated graphite potentiometric sensors based on 1-phenyl-3-pyridin-2-yl-thiourea for selective determination of iron (III), Electrochimica Acta, 55: 6724-6730.

[144] Zamani HA, et al., (2011), Quantitative Monitoring of Terbium Ion by a $\mathrm{Tb}^{3+}$ Selective Electrode Based on a New Schiff's Base, Materials Science and Engineering: C, 31: 409413.

[145] Álvarez-Romero GA, et al., (2010), Development of a Chloride Ion-Selective Solid State Sensor Based on Doped Polypyrrole-Graphite-Epoxy Composite, Electroanalysis, 22: 1650-1654. 
[146] Zahran EM, et al., (2009), Triazolophanes: A New Class of Halide - Selective Ionophores for Potentiometric Sensors, Analytical Chemistry, 82: 368-375.

[147] Kang Y, et al., (2010), Development of a Fluoride-Selective Electrode based on Scandium (III) Octaethylporphyrin in a Plasticized Polymeric Membrane, Bulletin of the Korean Chemical Society, 31: 1601-1608.

[148] Liang RN, Song DA, Zhang RM, and Qin W, (2010), Potentiometric Sensing of Neutral Species Based on a Uniform Sized Molecularly Imprinted Polymer as a Receptor, Angewandte Chemie, 122: 2610-2613.

[149] Madunić D, Sak-Bosnar M, and Matešić-Puač R, (2011), A New Anionic Surfactant Sensitive Potentiometric Sensor with a Highly Lipophilic Electroactive Material, International Journal of Electrochemical Science, 6: 240-253.

[150] Washe AP, Macho S, Crespo GA, and Rius FX, (2010), Potentiometric Online Detection of Aromatic Hydrocarbons in Aqueous Phase Using Carbon Nanotube - Based Sensors, Analytical Chemistry, 82: 8106-8112.

[151] Zhuiykov S, Kats E, and Marney D, (2010), Potentiometric Sensor Using Sub - Micron $\mathrm{Cu}_{2} \mathrm{O}$ - Doped $\mathrm{RuO}_{2}$ Sensing Electrode with Improved Antifouling Resistance, Talanta, 82: 502-507.

[152] Girish Kumar K, Augustine P, and John S, (2010), Novel potentiometric sensors for the selective determination of domperidone, Journal of applied electrochemistry, 40: 65-71.

[153] Abounassif A, Al-Hadiya BM, and Mostafa GAE, (2010), PVC Matrix Membrane Sensors for Potentiometric Determination of Arecoline, Instrumentation Science and Technology, 38: 165-177.

[154] Alizadeh T and Akhoundian M, (2010), A novel potentiometric sensor for promethazine based on a molecularly imprinted polymer (MIP): The role of MIP structure on the sensor performance, Electrochimica Acta, 55: 3477-3485.

[155] Vlascici D, et al., (2010), Manganese (III) Porphyrin - Based Potentiometric Sensors for Diclofenac Assay in Pharmaceutical Preparations, Sensors, 10: 8850-8864.

[156] Killard AJ and Smyth MR (2006 ), Electrochemical Immunosensors, In Grimes CA, et al.s Encyclopedia of Sensors, American Scientific Publishers, Pennsylvania:

[157] $\mathrm{Xu} \mathrm{H}$, et al., (2008), Ultrasensitive Voltammetric Detection of Trace Lead (II) and Cadmium (II) Using MWCNTs Nafion/Bismuth Composite Electrodes, Electroanalysis, 20: 2655-2662.

[158] Zima J, Švancara I, Barek J, and Vytřas K, (2009), Recent Advances in Electroanalysis of Organic Compounds at Carbon Paste Electrodes, Critical Reviews in Analytical Chemistry, 39: 204-227.

[159] Shang F, et al., (2009), Selective Nanomolar Detection of Dopamine Using a Boron Doped Diamond Electrode Modified with an Electropolymerized Sulfobutylether - $\beta$ Cyclodextrin - Doped Poly (N - Acetyltyramine) and Polypyrrole Composite Film, Analytical chemistry, 81: 4089-4098. 
[160] Yan J, et al., (2008), An Electrochemical Sensor for 3, 4 - Dihydroxyphenylacetic Acid with Carbon Nanotubes as Electronic Transducer and Synthetic Cyclophane as Recognition Element, Chemical Communications: 4330-4332.

[161] Siangproh W, Dungchai W, Rattanarat P, and Chailapakul O, (2011), Nanoparticle Based Electrochemical Detection in Conventional / Miniaturized Systems and their Bioanalytical Applications: A Review, Analytica Chimica Acta, 690: 10-25.

[162] Guo S and Wang E, (2011), Noble metal nanomaterials: Controllable synthesis and application in fuel cells and analytical sensors, Nano Today, 6: 240-264.

[163] Ispas CR, Crivat G, and Andreescu S, (2012), Review: Recent Developments in Enzyme-Based Biosensors for Biomedical Analysis, Analytical Letters, 45: 168-186.

[164] Moyo M, Okonkwo JO, and Agyei NM, (2012), Recent Advances in Polymeric Materials Used as Electron Mediators and Immobilizing Matrices in Developing Enzyme Electrodes, Sensors, 12: 923-953.

[165] Atta NF, El-Kady MF, and Galal A, (2010), Simultaneous determination of catecholamines, uric acid and ascorbic acid at physiological levels using poly (Nmethylpyrrole)/Pd-nanoclusters sensor, Analytical Biochemistry, 400: 78-88.

[166] Gholivand MB and Amiri M, (2009), Preparation of Polypyrrole/Nuclear Fast Red Films on Gold Electrode and Its Application on the Electrocatalytic Determination of Methyl-dopa and Ascorbic Acid, Electroanalysis, 21: 2461-2467.

[167] Zachek MK, et al., (2009), Simultaneous Decoupled Detection of Dopamine and Oxygen Using Pyrolyzed Carbon Microarrays and Fast - Scan Cyclic Voltammetry, Analytical Chemistry, 81: 6258-6265.

[168] Noroozifar M, Khorasani-Motlagh M, and Taheri A, (2010), Preparation of Silver Hexacyanoferrate Nanoparticles and its Application for the Simultaneous Determination of Ascorbic Acid, Dopamine and Uric Acid, Talanta, 80: 1657-1664.

[169] Rastakhiz N, Kariminik A, Soltani-Nejad V, and Roodsaz S, (2010), Simultaneous Determination of Phenylhydrazine, Hydrazine and Sulfite Using a Modified Carbon Nanotube Paste Electrode, International Journal of Electrochemical Science, 5: 12031212.

[170] Ensafi AA and Karimi-Maleh H, (2010), Modified multiwall carbon nanotubes paste electrode as a sensor for simultaneous determination of 6-thioguanine and folic acid using ferrocenedicarboxylic acid as a mediator, Journal of Electroanalytical Chemistry, 640: 75-83.

[171] Ghorbani-Bidkorbeh F, Shahrokhian S, Mohammadi A, and Dinarvand R, (2010), Simultaneous voltammetric determination of tramadol and acetaminophen using carbon nanoparticles modified glassy carbon electrode, Electrochimica Acta, 55: 27522759 .

[172] Kalimuthu P and John SA, (2010), Simultaneous Determination of Ascorbic Acid, Dopamine, Uric Acid and Xanthine Using a Nanostructured Polymer Film Modified Electrode, Talanta, 80: 1686-1691. 
[173] Ulubay S and Dursun Z, (2010), Cu Nanoparticles Incorporated Polypyrrole Modified GCE for Sensitive Simultaneous Determination of Dopamine and Uric Acid, Talanta, 80: 1461-1466.

[174] Ghasemi-Varnamkhasti M, et al., (2011), Electronic and bioelectronic tongues, two promising analytical tools for quality evaluation of non alcoholic beer, Trends in Food Science \& Technology, 22: 245-248.

[175] Woertz K, Tissen C, Kleinebudde P, and Breitkreutz J, (2010), Rational Development of Taste Masked Oral Liquids Guided by an Electronic Tongue, International Journal of Pharmaceutics, 400: 114-123.

[176] Riul Jr A, Dantas CAR, Miyazaki CM, and Oliveira Jr ON, (2010), Recent Advances in Electronic Tongues, Analyst, 135: 2481-2495.

[177] Gutiérrez M, et al., (2011), Application of an E-Tongue to the Analysis of Monovarietal and Blends of White Wines, Sensors, 11: 4840-4857.

[178] Gutiérrez M, et al., (2010), Hybrid electronic tongue based on optical and electrochemical microsensors for quality control of wine, Analyst, 135: 1718-1725.

[179] Chik H and Xu JM, (2004), Nanometric superlattices: non-lithographic fabrication, materials, and prospects, Materials Science and Engineering: R: Reports, 43: 103-138.

[180] Shi J, et al., (2004), Recent Developments in Nanomaterial Optical Sensors, TrAC Trends in Analytical Chemistry, 23: 351-360.

[181] Khanna VK, (2008), Nanoparticle - Based Sensors, Science, 58: 608-616.

[182] Ellis DI and Goodacre R, (2006), Metabolic fingerprinting in disease diagnosis: biomedical applications of infrared and Raman spectroscopy, Analyst, 131: 875-885.

[183] Naydenova I, Jallapuram R, Toal V, and Martin S, (2008), A Visual Indication of Environmental Humidity Using a Color Changing Hologram Recorded in a Self Developing Photopolymer, Applied Physics Letters, 92: 031109.

[184] Ahir SV, Huang YY, and Terentjev EM, (2008), Polymers with aligned carbon nanotubes: Active composite materials, Polymer, 49: 3841-3854.

[185] Wang J, Lin Q, Zhou R, and Xu B, (2002), Humidity Sensors Based on Composite Material of Nano - $\mathrm{BaTiO}_{3}$ and Polymer RMX, Sensors and Actuators B: Chemical, 81: 248-253.

[186] Novak BM, (1993), Hybrid Nanocomposite Materials - Between Inorganic Glasses and Organic Polymers, Advanced Materials, 5: 422-433.

[187] Selampinar F, et al., (1995), A Conducting Composite of Polypyrrole II. As a Gas Sensor, Synthetic metals, 68: 109-116.

[188] Patil D, Patil P, Seo YK, and Hwang YK, (2010), Poly (o - Anisidine) Tin Oxide Nanocomposite: Synthesis, Characterization and Application to Humidity Sensing, Sensors and Actuators B: Chemical, 148: 41-48.

[189] Barbadillo M, et al., (2009), Gold nanoparticles-induced enhancement of the analytical response of an electrochemical biosensor based on an organic-inorganic hybrid composite material, Talanta, 80: 797-802. 
[190] Mao S, et al., (2009), Ultrafast Hydrogen Sensing Through Hybrids of Semiconducting Single - Walled Carbon Nanotubes and Tin Oxide Nanocrystals, Physical Chemistry Chemical Physics (PCCP), 11: 7105-7110.

[191] Gopalan AI, et al., (2009), An electrochemical glucose biosensor exploiting a polyaniline grafted multiwalled carbon nanotube/perfluorosulfonate ionomer-silica nanocomposite, Biomaterials, 30: 5999-6005.

[192] Güell AG, Meadows KE, Unwin PR, and Macpherson JV, (2010), Trace voltammetric detection of serotonin at carbon electrodes: comparison of glassy carbon, boron doped diamond and carbon nanotube network electrodes, Physical Chemistry Chemical Physics (PCCP), 12: 10108-10114.

[193] Apetrei C, Apetrei IM, Saja JAD, and Rodriguez-Mendez ML, (2011), Carbon paste electrodes made from different carbonaceous materials: application in the study of antioxidants, Sensors, 11: 1328-1344.

[194] Radecka M., et al., Nanocrystalline TiO 2/SnO 2 composites for gas sensors, Journal of Thermal Analysis and Calorimetry: 1-6.

[195] Sung T.W. and Lo Y.L., (2012), Highly sensitive and selective sensor based on silicacoated CdSe/ZnS nanoparticles for $\mathrm{Cu}<\sup >2+</$ sup $>$ ion detection, Sensors and Actuators B: Chemical: 